Journal for ImmunoTherapy of Cancer

\title{
Peripherally-driven myeloid NFkB and IFN/ISG responses predict malignancy risk, survival, and immunotherapy regime in ovarian cancer
}

To cite: Sprooten J, Vankerckhoven A, Vanmeerbeek I, et al. Peripherally-driven myeloid NFkB and IFN/ISG responses predict malignancy risk, survival, and immunotherapy regime in ovarian cancer. Journal for ImmunoTherapy of Cancer 2021;9:e003609. doi:10.1136/ jitc-2021-003609

- Additional supplemental material is published online only. To view, please visit the journal online (http://dx.doi.org/10. 1136/jitc-2021-003609).

JS, AV, AC and ADG contributed equally.

Accepted 17 October 2021

Check for updates

(C) Author(s) (or their employer(s)) 2021. Re-use permitted under CC BY-NC. No commercial re-use. See rights and permissions. Published by BMJ.

For numbered affiliations see end of article.

Correspondence to Professor Abhishek D Garg; abhishek.garg@kuleuven.be

\section{ABSTRACT}

Background Tumors can influence peripheral immune macroenvironment, thereby creating opportunities for non-invasive serum/plasma immunobiomarkers for immunostratification and immunotherapy designing. However, current approaches for immunobiomarkers' detection are largely quantitative, which is unreliable for assessing functional peripheral immunodynamics of patients with cancer. Hence, we aimed to design a functional biomarker modality for capturing peripheral immune signaling in patients with cancer for reliable immunostratification.

Methods We used a data-driven in silico framework, integrating existing tumor/blood bulk-RNAseq or singlecell (sc)RNAseq datasets of patients with cancer, to inform the design of an innovative serum-screening modality, that is, serum-functional immunodynamic status (sFIS) assay. Next, we pursued proof-of-concept analyses via multiparametric serum profiling of patients with ovarian cancer (OV) with sFIS assay combined with Luminex (cytokines/soluble immune checkpoints), CA125-antigen detection, and whole-blood immune cell counts. Here, sFIS assay's ability to determine survival benefit or malignancy risk was validated in a discovery $(n=32)$ and/or validation $(n=699)$ patient cohorts. Lastly, we used an orthotopic murine metastatic OV model, with anti-OV therapy selection via in silico drug-target screening and murine serum screening via sFIS assay, to assess suitable in vivo immunotherapy options.

Results In silico data-driven framework predicted that peripheral immunodynamics of patients with cancer might be best captured via analyzing myeloid nuclear factor kappa-light-chain enhancer of activated B cells (NFKB) signaling and interferon-stimulated genes' (ISG) responses. This helped in conceptualization of an 'in sitro' (in vitro + in situ) sFIS assay, where human myeloid cells were exposed to patients' serum in vitro, to assess serum-induced (si)$\mathrm{NF \kappa B}$ or interferon (IFN)/ISG responses (as active signaling reporter activity) within them, thereby 'mimicking' patients' in situ immunodynamic status. Multiparametric serum profiling of patients with OV established that
sFIS assay can: decode peripheral immunology (by indicating higher enrichment of si-NFKB over si-IFN/ ISG responses), estimate survival trends (si-NFKB or siIFN/ISG responses associating with negative or positive prognosis, respectively), and coestimate malignancy risk (relative to benign/borderline ovarian lesions). Biologically, we documented dominance of pro-tumorigenic, myeloid si-NFKB response ${ }^{\mathrm{HIHH}}$ si-IFN/ISG response ${ }^{\mathrm{LOW}}$ inflammation in periphery of patients with OV. Finally, in an orthotopic murine metastatic OV model, sFIS assay predicted the higher capacity of chemo-immunotherapy (paclitaxelcarboplatin plus anti-TNF antibody combination) in achieving a pro-immunogenic peripheral milieu (si-IFN/ISG response ${ }^{\mathrm{HIHH}}$ Si-NFKB response ${ }^{\text {LOW }}$, which aligned with high antitumor efficacy.

Conclusions We established sFIS assay as a novel biomarker resource for serum screening in patients with OV to evaluate peripheral immunodynamics, patient survival trends and malignancy risk, and to design preclinical chemo-immunotherapy strategies.

\section{BACKGROUND}

Tumor-level proteogenomic or cellular immunobiomarkers have been crucial for the recent progress in immuno-oncology. ${ }^{1-3}$ However, such biomarkers are not necessarily robust at capturing the nature of systemic immune macroenvironment. ${ }^{45}$ This is essential because cancer is capable of restructuring the composition and functions of both local (tumor) as well as global (e.g., peripheral) immune processes that ultimately influence immunotherapy outcomes. ${ }^{5-7}$ Thus, characterization of novel non-invasive biomarkers detectable in patient periphery (serum, plasma, or blood) is highly important. ${ }^{8-10}$ Recently, several peripheral biomarkers like specific cytokines or chemokines, and/or immune cell-subsets have been assessed in 
immuno-oncology. ${ }^{11}{ }^{12}$ But this progress is still limited, as several of these biomarkers are either unreliable and/or their contextual functionalities are insufficiently known. For example, most cytokines/chemokines are not independent prognostic biomarkers and tend to be unreliable for rapid screening. ${ }^{1314}$ Although several multiplex techniques are available for high-density cytokine or chemokine screening, these advancements have not entirely overcome above immunological and clinical limitations. ${ }^{15}$

Multiplexed serum biomarker assays have a largely quantitative approach, which creates a hurdle in estimating the patient serum's immunome (i.e., a sum of all immunological functions), since such immunome mapping requires both quantitative and qualitative (functional) assessments. However, the quantity of a particular cytokine is not linearly associated with its biological functions. ${ }^{16}$ Moreover, patient serum tends to be a complex mixture of immunostimulatory, immunosuppressive and homeostatic cytokines/factors. ${ }^{17}$ Thus, factors with contradictory or complementary immune functions may simultaneously coexist in the serum. Accordingly, multiparametric detection of all these factors frequently ends up creating a problematic situation for serum immunome mapping, that is, detection of contradictory (functional or prognostic) trends for cytokines/factors, which eliminates concise immunological interpretations. While efforts are being made to overcome these limitations via application of advanced bioinformatics, such approaches tend to fail in predicting dynamic signaling-trajectories that distinguish cytokine responses. ${ }^{18}$

Currently in the field of immune biomarkers, reliable analysis of functional immunodynamics of patients' serum is a poorly studied topic. Considering the high patient-to-patient immune variations, immunodynamic biomarkers are prerequisite for reliable immunostratification and precision immunotherapy. ${ }^{15}$ To address this critical challenge, we conceptualized an innovative immunodynamic biomarker assay whose design was guided by a patient data-driven framework. The overall objective of this in silico framework was to use advanced computational immunology to create tangible hypotheses in terms of best immune-signaling pathway(s), type of immune cell, and suitable cancer type to prioritize, for the designing and the validation of the assay. This in silico framework revealed that the top two inflammatory pathways most likely to be induced by the serum of patients with cancer were myeloid nuclear factor kappa-light-chain enhancer of activated B cells (NFKB) or myeloid interferon-stimulated response element (ISRE) signaling (involving activation of interferon-stimulated genes (ISGs)). Based on this, we strategized a 'first-in-class' serum-functional immunodynamic status (sFIS) assay, wherein in vitro assessment of serum-induced (si)-NFKB and si-interferon (IFN) or ISG responses in human myeloid cells was supposed to 'mimic' in situ peripheral immune-status of the patients. Herein, we report sFIS assay's efficient use in patients with ovarian cancer (OV) to decode complex peripheral immunodynamics, estimate survival trends, perform patient immunostratification, and coestimate malignancy risks. Finally, through preclinical experiments, we also demonstrate that the sFIS assay can help design novel chemo-immunotherapy regime. For complete details on our study design and workflow, please refer to online supplemental figure $\mathrm{S} 1$.

\section{METHODS}

\section{Patient description and clinical analyses}

Patients diagnosed with an ovarian mass were enrolled in two prospective studies (OV-IMM-2014 and TRANSIOTA) and in an umbrella trial between June 2014 and June 2019. For this study, sera from OV-IMM-2014/ umbrella trial were part of the 'discovery cohort', whereas sera from TRANS-IOTA were part of the 'validation cohort'. OV-IMM-2014/umbrella patients were enrolled exclusively at University Hospitals (UZ) Leuven (Belgium). TRANS-IOTA was a European consortium study recruiting in Rome (Italy: Fondazione Policlinico Universitario Agostino Gemelli, Istituto di Ricovero e Cura a Carattere Scientifico), Prague (Czech Republic: Charles University), Genk (Belgium: Ziekenhuis Oost-Limburg), London (UK: Queen Charlotte's and Chelsea Hospital, Imperial College), Milan (Italy: National Cancer Institute of Milan) and UZ Leuven (Belgium). OV-IMM-2014/ umbrella recruited only patients with primary invasive OV (see online supplemental table 1 for patient details), whereas TRANS-IOTA recruited patients with ovarian masses at diagnosis (see online supplemental table 2 for patient details). All patients underwent surgery. Based on subsequent histopathological examination, patients were categorized as having a benign cyst, a borderline tumor, an invasive OV, or a metastatic tumor to the ovary. Exclusion criteria for all studies included women with active therapy for non-OV at the point of inclusion, presence of immune disease, treatment with immunomodulators, pregnancy, age $<18$ years, surgery of the suspected mass elsewhere before inclusion, and infectious serology (e.g., HIV and hepatitis B/C). Serum was obtained from all patients, as described previously. ${ }^{19}{ }^{20}$ Analyses for serum-associated carbohydrate antigen 125 (CA125) and white blood cell counts (absolute counts for lymphocytes, monocytes, neutrophils, and platelets) were part of standard clinical analyses, and these data were retrieved from the hospitallevel patient files. Of note, in some cases due to practical (e.g., unavailability of data in the central hospital database) or technical (e.g., not enough sample volumes for profiling or mismatched sampling timepoints) reasons, certain CA125 or white blood cell counts were not available for every sample.

\section{Standardization of sFIS assay}

THP1 dual cells (Invivogen) featuring the secreted luciferase reporter gene, under the control of an ISG54 minimal promoter in conjunction with five IFN-stimulated response elements (ISREs, under zeocin antibiotic selection), and secreted embryonic alkaline phosphatase 
(SEAP) reporter gene, driven by an IFN- $\beta$ minimal promoter fused to five copies of the NFKB consensus transcriptional response element and three copies of the c-Rel binding site (under blasticidin-antibiotic selection), were used. These cells were cultured at $37^{\circ} \mathrm{C}$ under $5 \% \mathrm{CO}_{2}$ in RPMI-1640 containing $2 \mathrm{mM}$ L-glutamine, $25 \mathrm{mM} \mathrm{4-(2-}$ hydroxyethyl)-1-piperazineethanesulfonic acid (HEPES), 100 units/mL penicillin, $100 \mu \mathrm{g} / \mathrm{L}$ streptomycin, 100 $\mu \mathrm{g} / \mathrm{mL}$ normocin and $10 \%$ heat-inactivated fetal bovine serum (FBS). For selection of dual reporter expressing cells, the cells were cultured with $10 \mu \mathrm{g} / \mathrm{mL}$ blasticidin and $100 \mu \mathrm{g} / \mathrm{mL}$ zeocin after two passages, after thawing. For standardization and validation exercises, these THP1 cells were incubated for $48 \mathrm{~h}$ with lipopolysaccharide (LPS), 5'ppp-dsRNA, 2,3-cGAMP, IFN- $\alpha 2 \mathrm{~b}$ (Invivogen) or TNF, Trail, IFN $\beta / \gamma$, interleukin (IL)-1 $\beta$, IL-6, IL-10, IL-18 (Peprotech) or recombinant versions of programmed cell death protein 1 (PD-1), PDL-1 and T-cell immunoglobulin and mucin domain 3 (TIM3) (Biolegend). Luciferase activity was checked in media by adding $50 \mu \mathrm{L}$ of Quanti-Luc (Invivogen) to $100 \mu \mathrm{L}$ of (separately recovered) THP1 media. Bioluminescence was examined for $100 \mathrm{~ms}$ immediately after Quanti-Luc addition by microplate reader (Biotek). SEAP activity was checked in media by adding $100 \mu \mathrm{L}$ of Quanti-Blue (Invivogen) to $100 \mu \mathrm{L}$ of (separately recovered) THP1 media. Absorbance was examined at an optical density of $655 \mathrm{~nm}, 4-8 \mathrm{~h}$ after Quanti-Blue addition by microplate reader (Biotek).

\section{Human serum screening via sFIS assay}

For human serum analysis, THP1 dual cells (Invivogen) were seeded in a 96-well plate at a density of 30000 cells/ well in $100 \mu \mathrm{L}$ media. After $24 \mathrm{~h}$, THP1 cells were treated with $100 \mu \mathrm{L}$ of normal human serum (pooled from several normal human individuals, Sigma-Aldrich) as baseline for our assay, or with patient serum for $24 \mathrm{~h}$. As a positive control, THP1 cells were treated with $1000 \mathrm{ng} / \mathrm{mL}$ LPS. Luciferase (bioluminescence) or SEAP (colorimetric) activity in the media was assessed as described previously.

\section{SUPPLEMENTARY METHODS}

Please refer to the online supplemental methods document for details on computational/bioinformatic analyses, Luminex sera-analyses, OV mouse model, murine therapeutic treatment procedures, murine serumscreening via sFIS assay and statistical analyses and data visualization.

\section{RESULTS}

\section{Major peripheral immunobiomarkers in oncology} predominantly engage NFKB or IFN/ISG responses

Cytokines induce an array of different downstream immune pathways, for example, NFkB response, IFN-induced ISG response, AP1 signaling, SMAD signaling, TRADD/FADD signaling, FOXO signaling or p38/ERK signaling. ${ }^{21-23}$ However, to design a precise serum-screening functional assay (online supplemental figure S1), we needed to focus on immune pathways dominantly engaged by majority of cytokines/immune factors in oncological context. To enable such consolidation exercise in an unbiased manner, we pursued a twostep bioinformatic process. In step 1 , we delineated the most frequently screened peripheral (oncological) immunobiomarkers using an automated MELODI literaturemining algorithm that extracts potential gene/protein identities between any two (prespecified) biomedical concepts. ${ }^{24}$ Herein, MELODI was executed to identify the most frequently occurring genes / proteins extracted from PubMed literature (1950-2017) simultaneously relevant for two concepts, that is, 'serum of patients with cancer' and 'immunological factors' (defined using precise string of keywords; see online supplemental methods for details). This exercise identified 54 immunological, inflammatory, and/or haematopoietic factors most frequently assessed as peripheral oncological biomarkers (online supplemental figure S2A). In step 2, we used these 54 major peripheral immunobiomarkers to identify the dominant downstream immune pathways and associated transcriptional programs. A computational analysis enriching for transcription factor (TF) targets via top three REACTOME biological pathways linked to the aforementioned 54 immunobiomarkers (co-delineated along with top hallmark gene sets to provide context) found that inflammation or effector immunity operating via either an $\mathrm{NF \kappa B}$ response (NFKB/REL) or an IFN/ ISG response (ISRE-binding TFs, IRF and IRF1/8) were the most dominantly enriched downstream pathways/ TF classes (online supplemental figure S2B). Thus, the major oncological peripheral immunobiomarkers dominantly engaged the NFKB or IFN/ISG responses.

\section{Tumor and peripheral immune cells co-enrich differential NFKB or ISG response patterns}

Tumors can, directly or indirectly, influence the differential enrichment of some peripheral cytokines. ${ }^{525}$ Hence, we interrogated: (1) does a co-enrichment exist for NFKB or ISG responses in immune cells across both tumor and periphery; and (2) which immune cells typically capture this co-enrichment (especially in periphery)? The former was vital to prove the suitability of a data-driven framework reliant on the multiomics tumor datasets of patients with cancer, while the latter was central to delineate most suitable immune cell type. Both steps were necessary to reveal our assay's target cancer type(s) and immune cell type.

To enable multiomics analyses, we used a validated genetic NFKB signaling signature ${ }^{26}$ and created a consensus genetic ISG response signature. The ISG response signature was a consensus between three validated genetic signatures induced downstream of IFN- $\alpha,{ }^{27}$ $\operatorname{IFN} \beta^{28}$ or IFN $\gamma^{29}$ We prioritized a consensus signature because a computational analysis (driven by the Interferome database of curated experimental datasets ${ }^{30}$ ) investigating the overlaps of downstream genes/ISGs 
regulated by all three IFN cytokine-types (I, II or III) showed that there was a considerably high sharing of ISGs induced downstream of all three IFN cytokine types, such that $\geq 85 \%$ ISGs (irrespective of which signature was used) were either overlapping between all three IFN cytokine types or between type I/II IFNs (online supplemental figure S3). Thus, to avoid unnecessary redundancy, ${ }^{31}$ we used a consensus ISG response signature.

Next, to assess the systemic inflammatory circuit on the level of NFKB or IFN/ISG responses, we analyzed an existing scRNAseq map that profiled tumor-infiltrating and (matched) blood-derived immune cells (procured from patients with renal cell or large cell neuroendocrine carcinoma), ${ }^{32}$ with the aforementioned NFKB or ISG response signatures (figure 1A,B). Across both blood and tumor immune cells, NFKB response signature was more strongly enriched than ISG response signature, such that myeloid cells particularly co-enriched these contrasting patterns in both tumors and blood (figure $1 \mathrm{~A}, \mathrm{~B}$ and online supplemental figure $\mathrm{S} 4 \mathrm{~A}, \mathrm{~B})$. Overall, NFкB response signature had the highest enrichment in blood myeloid cells (online supplemental figure S4A), while in tumors, only B cells, $\mathrm{CD}^{+} \mathrm{T}$ cells and Tregs had higher NFKB response signature than myeloid cells (online supplemental figure S4B). IFN/ISG response signature was relatively better enriched in $\mathrm{T}$ cells (online supplemental figure S4A,B). Nevertheless, for myeloid cells, it is necessary to consider that NFKB (subset 3) and ISG (subset 4) response signatures were enriched in different subsets of blood myeloid cells (figure 1A,B) such that both in blood (online supplemental figure S4C) or tumor (online supplemental figure S4D) myeloid cells, there was negative/null correlation between NFKB and ISG response signature enrichment. Overall, this implied a differential co-enrichment of NFKB and IFN/ISG response circuits between the tumor and the periphery, particularly represented by myeloid cells in both compartments.

\section{Tumoral immunodynamics of NFKB or ISG responses predict survival of patients with cancer}

Based on the aforementioned observations, we used The Cancer Genome Atlas (TCGA) tumor datasets for creating a data-driven framework (essential for our assay's designing) driven by the qualitative and prognostic behavior of NFKB/ISG response patterns. Herein, we selected 12 TCGA datasets (spanning $>5000$ patients with cancer; see online supplemental methods for selection strategy) with diverse solid tumors typically showing either immunotherapy responsiveness (i.e., lung cancer (LUAD/LUSC), head and neck cancer (HNSC), bladder cancer, renal cell cancer, and liver cancer (LIHC)), or immunotherapy resistance $(\mathrm{OV}$, endometrial cancer, sarcoma, breast cancer, pancreatic cancer, and cervical cancer (CESC) ). ${ }^{33}$ Interestingly, nearly half of the 54 major oncological (peripheral) immunobiomarkers were not sufficiently expressed in these 12 tumor types (online supplemental figure S5A). Moreover, the 25 highly expressed immunobiomarkers did not create distinct clusters that could clearly differentiate immunotherapy responsive from non-responsive cancer types (online supplemental figure S5A). Also, these immunobiomarkers exhibited a variable cancer type-dependent prognostic impact on overall survival (OS) (online supplemental figure S5B).

Next, we attempted to understand whether the more simplified 'dynamics' of the binary NFKB/ISG responses may better summarize a general inflammatory status than the aforementioned heterogenous patterns. To address this, we first derived the median expression and median hazard ratios (HRs) for each of the 25 highly expressed immunobiomarkers across all 12 cancer types and applied dimensionality reduction (i.e., Principal component analysis or PCA) (online supplemental figure S5 and figure 1C). This consolidated two distinct clusters, that is, a cluster of immunobiomarkers with negative prognostic impact and those with positive prognostic impact (figure 1C). Next, we annotated the aforementioned genes as either NFKB target genes (NTGs) ${ }^{35} 36$ or ISGs based on available literature. ${ }^{30}$ This revealed that the negative prognostic cluster was associated with a disbalance in these modalities, favoring NFKB signaling (NTGs $>$ ISGs); whereas positive prognostic cluster had a relatively better balance (NTGs $\approx$ ISGs) (figure 1C). Thus, a disbalance in tumoral immunodynamic signaling that favors NFKB responses predicted shortened patient survival, whereas more balanced signaling between ISG/NFkB responses predicted prolonged patient survival.

\section{Ovarian tumor-linked myeloid milieu enriches for disbalanced NFKB/ISG responses}

Next, we estimated the prognostic impact of tumoral $\mathrm{NFKB} /$ ISG response signatures on patient OS in the aforementioned 12 TCGA cancer types to delineate which cancer types might best capture the contrasting interplay between NFKB (negative prognostic) and IFN/ISG (positive prognostic) responses (figure 1D,E). Herein, predominantly CESC or OV exhibited (statistically significant) contradictory prognostic impacts for NFKB and ISG response signatures (figure $1 \mathrm{D}, \mathrm{E}$ ). Similar trends were also observed for LIHC/HNSC. Herein for further investigation, we selected OV since it is associated with several clinically relevant diagnostic and prognostic peripheral biomarkers like CA125, HE4, or Ova1/Overa. ${ }^{37}$

Next, we explored whether the differential NFкB/ ISG response patterns observed in the aforementioned scRNAseq analyses were also applicable to OV, to ensure consistency in our data-driven framework. Henceforth, we pursued analyses of an existing scRNAseq dataset of a patient with OV (see online supplemental methods for selection strategy). Within OV tumor-derived cancer, immune and stromal cells (figure $1 \mathrm{~F}$ ), ${ }^{38}$ analyses for $\mathrm{NFKB}$ (figure 1G) or ISG (figure 1H) response signatures showed that myeloid cells indeed enriched for higher NFKB response signature than the ISG response signature, with the NFkB response signature being more highly enriched in myeloid cells than other immune cells 


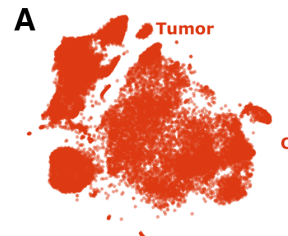

A

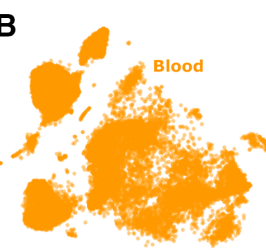

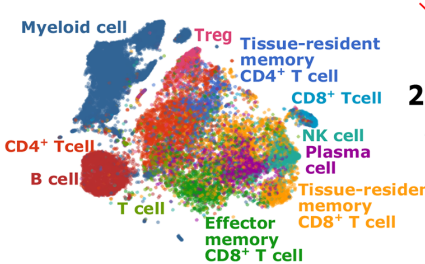

memory

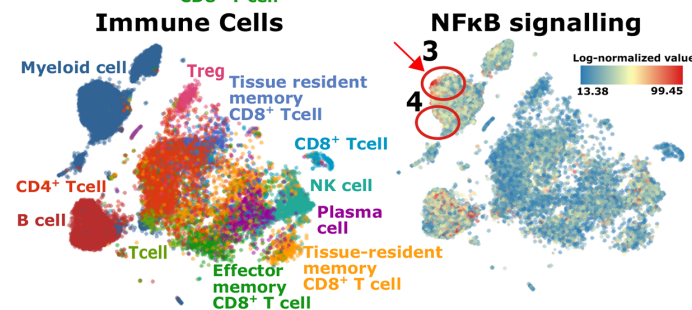

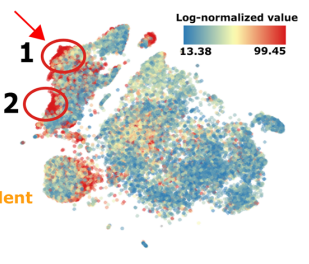

NFKB signalling

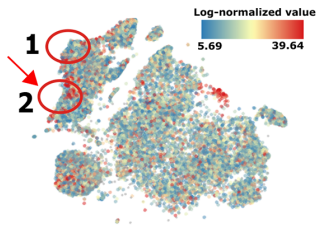

C

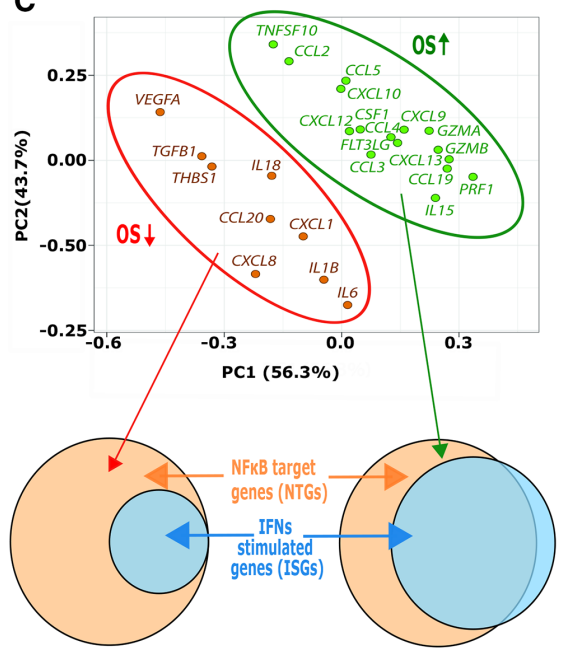

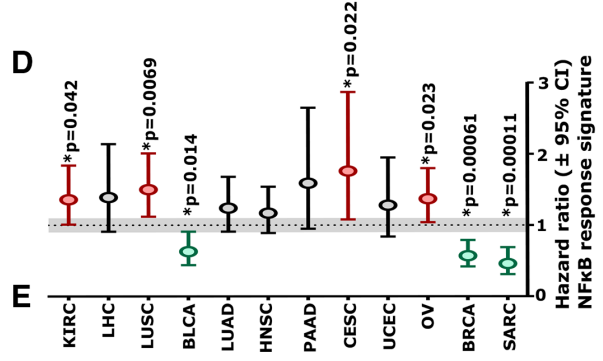

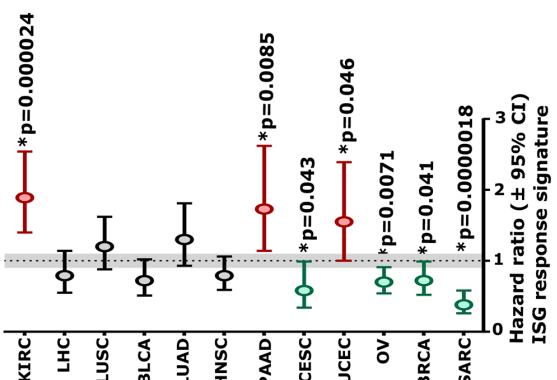

F

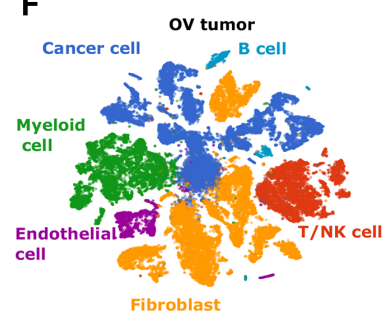

G

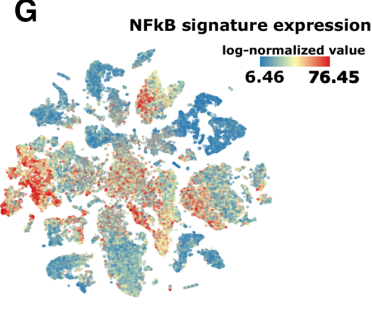

$\mathrm{H}$

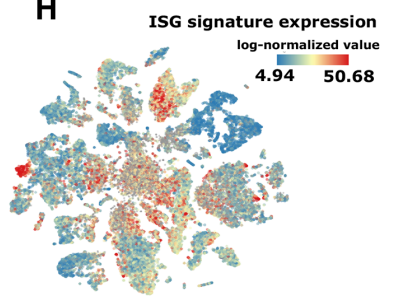

Figure 1 Interrogation of the tumour derived bulk-RNAseq or tumour/blood single-cell (sc)-RNAseq data of patients with cancer. $(A, B)$ The t-distributed stochastic neighbor embedding (tSNE) visualizations of indicated immune cell types, from scRNAseq data from patients with renal cell and large cell neuroendocrine carcinoma $(n=4$ patients in total) (derived from GSE139555) isolated from tumor tissue (A) or peripheral blood (B). These immune cells were further colored for NFKB and IFN/ ISG response gene-signature levels. Herein, the arrows highlight main immune populations with overlaps between tumor and blood for these signatures, and the circles indicate different myeloid cell subsets. (C) PCA analyses of median expression for each gene across cancer types (from online supplemental figure S5A) and median HR values (from online supplemental figure $\mathrm{S} 5 \mathrm{~B})$. Venn diagram represents the portion of $\mathrm{NF} \kappa \mathrm{B}$ and ISGs in each cluster. (D,E) Visualization of the hazard ratio $(\mathrm{HR}) \pm 95 \%$ confidence interval $(\mathrm{Cl})$ for the impact of expression of NFKB signaling gene signature (D) or ISG signaling gene signature (E), for the indicated TCGA cancer datasets wherein the signature expression cut-off for binary (high vs low expression) patient stratification was based on best-performing threshold principle for OS of indicated TCGA patients with cancer (LIHC, $\mathrm{n}=371$; PAAD, $n=177$; LUSC, $n=501$; LUAD, $n=513$; HNSC, $n=500 ;$ CESC, $n=304$; BLCA, $n=405$; UCEC, $n=543 ;$ OV, $n=374 ;$ SARC, $\mathrm{n}=259$; BRCA; $\mathrm{n}=1090$; KIRC; $\mathrm{n}=530$ ) (Mantel-Cox test, $\left.{ }^{*} \mathrm{p}<0.05\right)$. ( $\left.\mathrm{F}-\mathrm{H}\right)$ tSNE visualizations of indicated immune cell-types, from scRNAseq data from patients with OV ( $n=5$ patients) (derived from GSE146026) isolated from tumor tissue. These immune cells were furthered colored for NFKB (G) and ISG signature expression levels (H). BLCA, bladder cancer; CESC, cervical cancer; HNSC, head and neck cancer; IFN, interferon; ISG, interferon-stimulated gene; KIRC, renal cell cancer; LIHC, liver cancer; LUSC, lung cancer; LUAD, lung adenocarcinoma; NFאB, nuclear factor kappa-light-chain enhancer of activated B cells; OS, overall survival; OV, ovarian cancer; PAAD, pancreatic cancer; SARC, sarcoma; BRCA, breast cancer;TCGA, The Cancer Genome Atlas; UCEC, endometrial cancer. 
(online supplemental figure S6A). Of note, while the ISG response signature was largely expressed by myeloid, endothelial or fibroblast cells, the primary source of IFNcytokine gene (IFNG) was T/NK cells (online supplemental figure S6B). Moreover, within the myeloid cells, there was negative/null correlation between NFkB versus ISG response signatures (online supplemental figure $\mathrm{S} 6 \mathrm{C})$. In line with this, we observed that indeed relatively non-associated myeloid cell subsets enriched for NFкB versus ISG response signatures (online supplemental figure S7A). Interestingly, a differential REACTOME pathway enrichment analysis between $\mathrm{NF} \kappa \mathrm{B}$ response signature ${ }^{\mathrm{HIGH}}$ versus ISG response signature ${ }^{\mathrm{HIGH}}$ myeloid cells (online supplemental figure S7B) showed that NFKB response $\mathrm{e}^{\mathrm{HIGH}}$ myeloid cells enriched for anti-inflammatory or immuno-regulatory pathways (e.g., prostaglandin/ IL-10 signaling, and RUNX1/FOXO/CD163 pathways), whereas ISG response ${ }^{\mathrm{HIGH}}$ myeloid cells enriched for proinflammatory/immunogenic pathways (e.g., antiviral/ IFN signaling and antigen presentation). Altogether, this showed that OV myeloid compartment enriches for disbalanced NFKB and ISG response dynamics, wherein the former has anti-inflammatory/immunoregulatory associations, while the latter has pro-inflammatory/ immunogenic associations.

\section{sFIS assay for testing the peripheral NFKB or IFN/ISG responses of patients with cancer}

The aforementioned data-driven framework informed us that the dominant peripheral immunodynamics in $\mathrm{OV}$ might be best captured by human myeloid cell-associated $\mathrm{NFKB}$ or IFN/ISG responses. However, transcriptomic or proteomic analyses of these signaling pathways' key components within circulating human myeloid cells would not be very throughput and may not capture the overall dynamism of these signaling modalities. To address this, we decided to investigate whether co-culturing human myeloid cells in the presence of serum samples from patients with OV and then assessing the myeloid cell's NFKB or IFN/ISG signaling as cellular assay output may capture peripheral immunodynamics in $\mathrm{OV}$, in a throughput and dynamic fashion. Hence, we conceptualized an innovative in sitro sFIS assay for in vitro assessment of patient sera-induced si-NFkB and si-IFN/ISG response in human myeloid THP1 cells (figure 2A). This involved a 96-well plate-based throughput screening method using THP1 myeloid cell line stably expressing two inducible reporter constructs encoding for (1) a LUCIA luciferase gene (coding for a secreted form of coelenterazineusing luciferase enzyme) under the direct control of an ISG54 minimal promoter linked to five copies of ISRE sequences; and (2) a SEAP reporter gene (coding for the SEAP enzyme, a placental alkaline phosphatase) under the control of an IFN- $\beta$ minimal promoter linked to three copies of c-REL binding site and five copies of the NFKB consensus transcriptional response element (figure 2A). Once these THP1 reporter cells sense factors/cytokines that engage the NFKB and/or IFN/ISG responses, they should secrete extracellular SEAP/LUCIA that, in presence of proper substrates, create colorimetric (SEAP) or bioluminescent (LUCIA) readouts (figure 2A).

Accordingly, THP1 reporter cells exposed to patternrecognition receptor agonists like LPS (a TLR4 agonist), 5'ppp-dsRNA bound to a transfection reagent LysoVec (a RIG-I agonist), or 2'3'-cGAMP (a STING agonist) differentially stimulated the NFKB (figure 2B) and/or IFN/ISG (figure 2C) responses. Similarly, treatment with diverse human recombinant cytokines induced either NFKB response (like TNF or IL1 $\beta$ ) (figure 2D) or IFN/ISG response (like IFN- $\beta$, IFN- $\alpha$, or IFN- $\gamma$ ) (figure 2E). One of the emerging categories of serum immunobiomarkers in immuno-oncology is the soluble immune-checkpoint (e.g., TIM3 or PD-1/PD-L1) ${ }^{39}$ Hence, we exposed these reporter cells to human recombinant PD-1, TIM3, or PD-L1 proteins and observed that whereas they were in general incapable of substantially inducing either of the signaling programs, yet recombinant TIM3/PD-1 induced threshold levels of NFKB response (figure 2F,G). Thus, we hypothesized that a THP1 NFKB and IFN/ISG reporter myeloid cell-driven sFIS assay may sufficiently capture the downstream signaling impact of peripheral cytokines/ immune factors.

\section{Peripheral immunobiomarkers hint toward disbalanced NFкB and IFN/ISG responses in patients with OV}

To pursue a proof-of-concept for our sFIS assay approach, we aimed for a 'prospective retrospective' biomarker validation strategy, ${ }^{41}$ starting with a discovery cohort composed of clinical routine samples, that is, 98 archived serum specimens from randomly selected 32 patients with OV and random timepoints throughout the patient's disease course at UZ Leuven, Belgium (hereafter referred to as the UZL-CSI OV cohort). For patient characteristics, see online supplemental table 1 . The overall cumulative distribution of patient OS in the UZL-CSI OV cohort was not significantly different from the TCGA patients with $\mathrm{OV}$, although median OS was moderately towards the lower side (online supplemental figure S8A), indicating that our cohort had more aggressive OV tumors. This point was better indicated by a comparison of tumor stages III/IV, wherein our cohort had far more patients with stage IV relative to stage III OV, as compared with the TCGA OV cohort (online supplemental figure S8B). Importantly though, for validating immunobiomarkers, the UZL-CSI cohort was highly suitable since it provided the necessary immunological dynamism, on account of being composed of a diversity of pretreatment and on/ post-treatment sera samples (with chemo/targeted/ immunotherapies) (online supplemental figure S8C). Moreover, we also had several patients for which multiple time-series samples were available (online supplemental figure S8D) combined with variable distribution of tumor burden, estimated via serum CA125 levels (online supplemental figure S8E). The UZL-CSI cohort was composed of all four pathological responses (pRs) to therapy (online supplemental figure $\mathrm{S} 8 \mathrm{~F}$ ). 
A

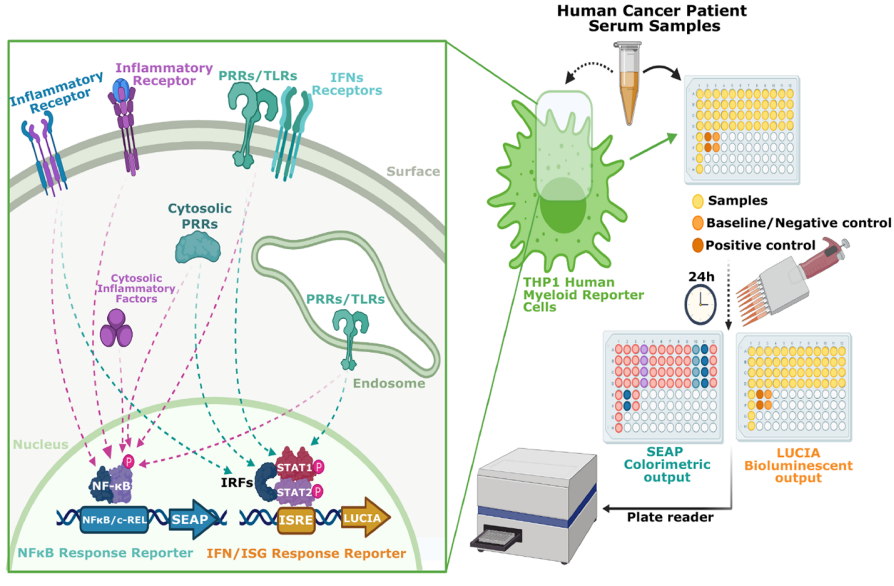

B

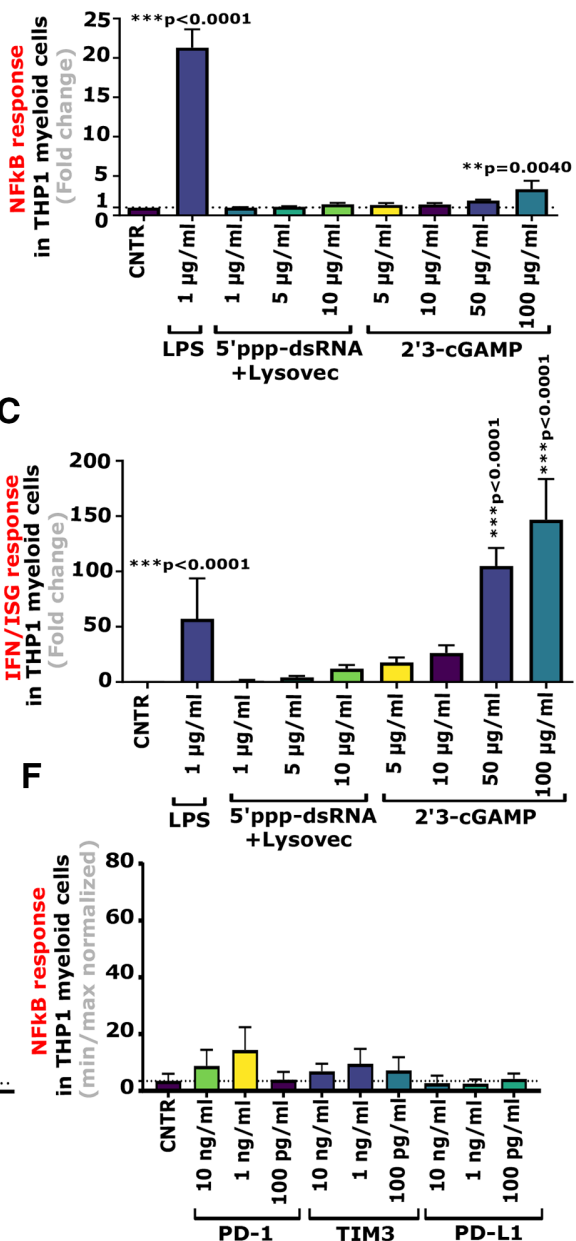

G

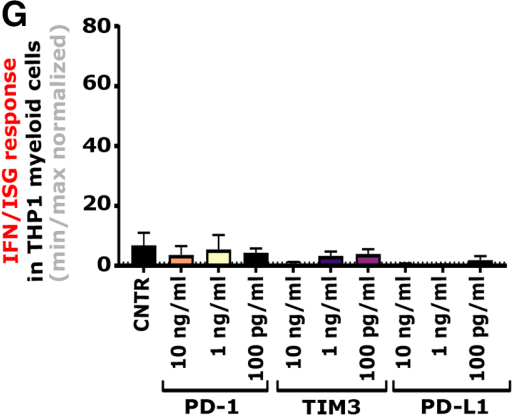

Figure 2 Standardization of SFIS assay. (A) Overview of the SFIS assay. THP1-dual reporter cells containing a reporter for NFKB and IFN/ISG response (see the Methods section) were stimulated with human serum of a patient with cancer, healthy donor serum (baseline) or LPS (positive control) for $24 \mathrm{~h}$. Subsequently, THP1 media were checked for LUCIA and SEAP activity. $(B, C)$ Bar graph of THP1-dual reporter cells, exposed to indicated concentrations of different agonists, reporting NFKB (B) or IFN/ISG (C) responses at $48 \mathrm{~h}$ post-treatment $(\mathrm{n}=3$, one-way analysis of variance with Fisher's Least Significant Difference (LSD) test; * $p<0.05)$. (D-G) Bar graph of THP1 dual reporter cells exposed to indicated concentrations of different cytokines $(D, E)$ or soluble immune checkpoints $(\mathrm{F}, \mathrm{G})$ reporting NFKB $(\mathrm{D}, \mathrm{F})$ or IFN/ISG $(\mathrm{E}, \mathrm{G})$ responses at $48 \mathrm{~h}$ post-treatment (min-max normalized, $n=4$, Kruskal-Wallis test; * $\mathrm{p}<0.05$ ). cGAMP, cyclic guanosine monophosphate-adenosine monophosphate; IFN, interferon; IL, interleukin; IRF, interferon regulatory factor; ISG, interferon-stimulated gene; ISRE, interferon-stimulated response element; LPS, lipopolysaccharide; NFאB, nuclear factor kappa-light-chain enhancer of activated B cells; PD-1, programmed cell death protein 1; PDL-1, programmed cell death-ligand 1; PRR, pattern recognition receptor; TIM3, T-cell immunoglobulin and mucin domain 3; TNF, tumor necrosis factor; TLR, toll-like receptor; TRAIL, TNF-related apoptosis-inducing ligand; SEAP, secreted embryonic alkaline phosphatase; sFIS, serumfunctional immunodynamic status; -STAT, signal transducer and activator of transcription.

To immunologically benchmark this UZL-CSI cohort, relative to our TCGA observations, we pursued a Luminex assay-based multiplex profiling of various serum immunobiomarkers like NFKB target proteins (IL-10, IL-12p70,
PGF, CCL2, CCL22, VEGF-A, CCL5, and MMP9), IFNs or ISGs-coded proteins (IFN- $\alpha$, IFN- $\gamma$, CXCL10, and CCL11), and some pleiotropic factors (IL-6, latency-associated peptide/transforming growth factor- $\beta$ (LAP/TGF- $\beta$ ), 


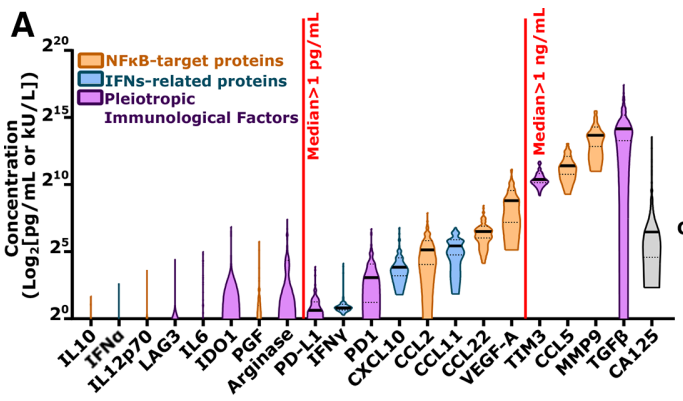

B
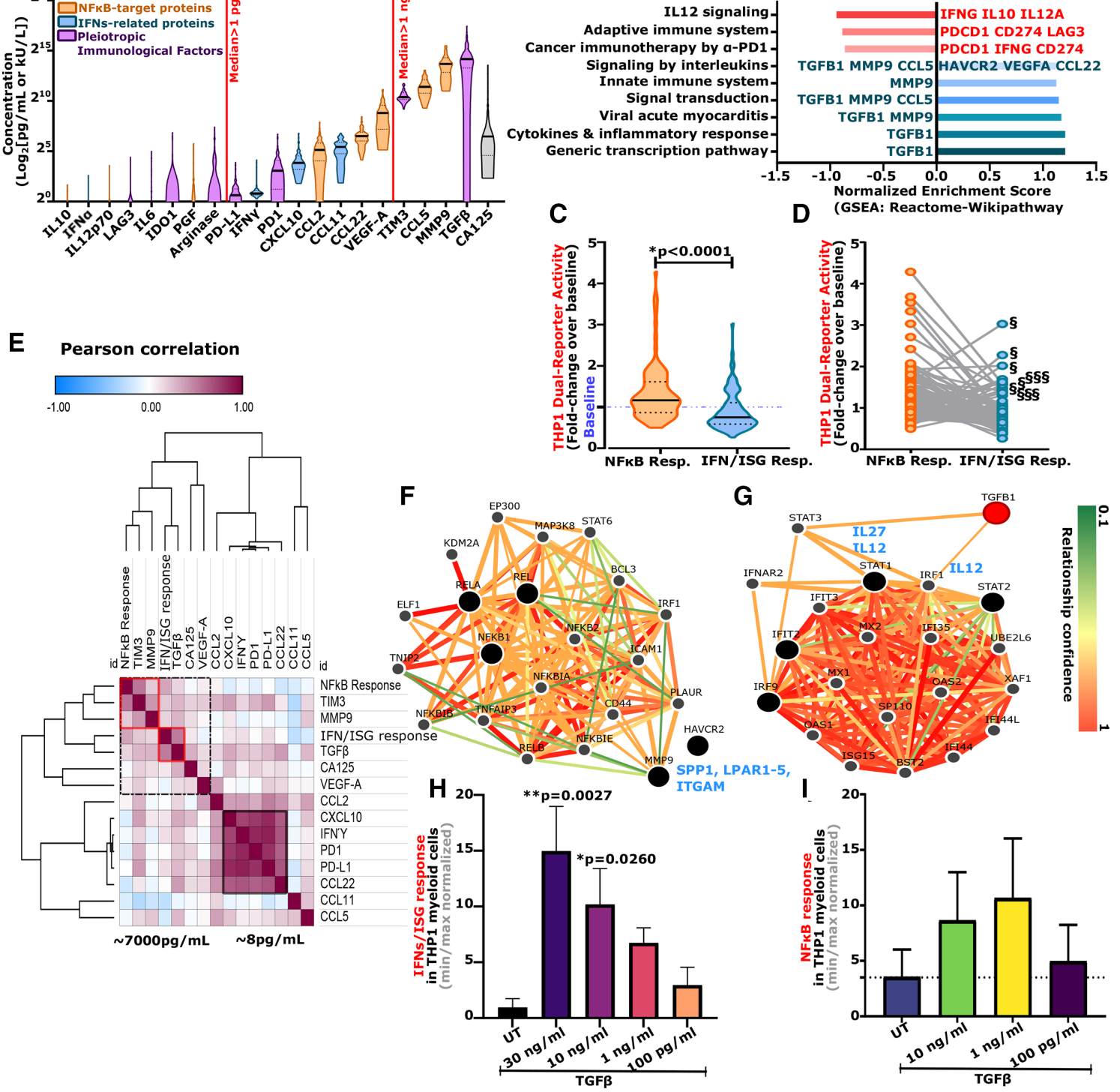

Figure 3 Immunological benchmarking and sFIS assay testing in discovery cohort of patients with OV. (A) Eligible serum samples from the UZL-CSI cohort were profiled for different immunological factors $(n=74)$ and CA125 $(n=95)$. Colors indicate association with categories mentioned in the legend. (B) REACTOME-Wikipathway GSEA based on concentrations of immunological biomarkers or CA125 (top 10 significance method). Violin plot (C) and sample-paired analysis (D) of UZL-CSI serum samples profiled for the si-NFKB or si-IFN/ISG response ( $n=96$, Wilcoxon matched-pairs signed-rank test; $\left.{ }^{*} p<0.05\right)$. ' $\S$ ' depicts serum samples wherein the si-IFN/ISG response values exceeded the si-NFKB response values. (E) Heatmap representation of Pearson correlation matrix of si-NFKB and si-IFN/ISG response, CA125 and immunological factors profiled from the serum of the UZL-CSI OV dataset $(n=74)$. ( $F, G)$ Network analyses of NFKB (F) or IFN/ISG (G) response activating transcription program. Genes in blue indicate immunologically relevant factors enumerated by the PID analyses. (H,I) Bar graph of THP1-dual reporter cells exposed to indicated concentrations of TGF- $\beta$ reporting IFN/ISG (H) or NFKB (I) responses at $48 \mathrm{~h}$ post-treatment $(n=3$, one-way analysis of variance corrected for false discovery rate via Benjamini, Krieger and Yekutieli test; $\left.{ }^{*} \mathrm{p}<0.05\right)$. CA125, carbohydrate antigen 125; GSEA, gene set enrichment analysis; IFN, interferon; ISG, interferon-stimulated gene; NFKB, nuclear factor kappa-light-chain enhancer of activated B cells; OV, ovarian cancer; PID, Pathway Interaction Database; sFIS, serum-functional immunodynamic status; si, serum-induced; UZL-CSI, UZ Leuven-Cell Stress Immunity cohort.

arginase), or soluble immune checkpoints (LAG3, IDO1, PD-L1, PD-1, and TIM3) (figure 3A). Serum-CA125 was also profiled to enable direct comparison with a clinical standard biomarker (figure 3A). This multiplexed analysis showed that sera of UZL-CSI patients with OV enriched for more NFKB-target proteins or pleiotropic factors, than IFNs/ISGs-coded proteins (figure 3A). Accordingly, a gene set enrichment analysis (GSEA)-based biological pathway enrichment (REACTOME/Wikipathways) computed on the aforementioned concentrations showed a significant positive enrichment for innate immune response or myeloid inflammation and a significant negative enrichment for adaptive or lymphoid pathways (figure 3B). These observations indirectly hinted toward 
a peripheral disbalance between NFkB target proteins and IFN/ISGs-related proteins in patients with OV.

\section{sFIS assay captures peripheral disbalance in si-NFKB and si- IFN/ISG responses}

We wondered whether our sFIS assay can faithfully capture the serum immunodynamics partly hinted by the aforementioned cytokines/factors. Remarkably, serum derived from these UZL-CSI patients with OV indeed induced significantly higher NFKB responses in the THP1 cells as compared with IFN/ISG responses (figure 3C). In fact, the median si-IFN/ISG responses were below our assay's baseline (i.e., normal human serum pooled from several healthy donors), thereby suggesting a tendency of sera of patients with OV to downregulate IFN/ISG responses. In fact, there were only 11 serum specimens (out of 98) that induced a higher fold change of si-IFN/ ISG responses than si-NFkB responses (figure 3D). These initial observations clearly outlined that our sFIS assay can faithfully capture the functional immunodynamics in sera of patients with OV, thereby more directly emphasizing the predominance of non-immunogenic inflammation in OV.

\section{si-NFKB response links to wound healing-like pro-metastatic signaling, while si-IFN/ISG response is proimmunogenic}

Next, we wished to gain some biological insights via integrated correlative and systems biology analyses of si-NFKB or si-IFN/ISG responses and serum immunobiomarkers. A correlation matrix between the major serum immunobiomarkers (median concentration $>1$ $\mathrm{pg} / \mathrm{mL}$ ), CA125 and sFIS assay responses showed the existence of two major clusters (figure 3E): (1) a very low concentration (median $\sim 8 \mathrm{pg} / \mathrm{mL}$ ) but very highly correlated, IFN $\gamma$-centered immunosuppressive cluster (co-recruiting soluble PD-1/PDL1); and (2) a very high concentration (median $\sim 7000 \mathrm{pg} / \mathrm{mL}$ ) but somewhat loosely correlated cluster, wherein two sub-clusters were formed by si-NFKB response and soluble TIM3/ MMP9 or si-IFN/ISGs response and LAP/TGF- $\beta$. To further understand these co-clustering patterns, we carried out immunological functional network analyses using genetic representatives. This revealed that the NFkB-relevant genes were associated with MMP9 (but not TIM3) via anti-inflammatory or prometastatic signaling modules ${ }^{42}{ }^{43}$ like wound healing facilitators (osteopontin-coding, SPP1 and integrin $\alpha$ M-coding $(I T G A M))^{4445}$ or protumorigenic lipid signaling (lysophosphatidic acid receptors-coding (LPAR1-5) $)^{46} 47$ (figure 3F). Interestingly, the IFN/ISG-relevant genes were associated with $T G F B 1$ via proinflammatory factors like IL-27, IL-12, STAT3 and IRF1 (figure 3G). This implicated a contextual immunogenic role for TGF- $\beta$. In fact, it has been reported that high concentration of TGF- $\beta$ can induce IFN/ISG responses. ${ }^{48}{ }^{49}$ Intriguingly, we indeed saw that treatment of THP1-reporter cells with high concentrations of TGF- $\beta$ (like concentrations recovered from OV-patients in UZL-CSI cohort) induced highly significant IFN/ISG (but not NFkB) responses (figure $3 \mathrm{H}, \mathrm{I}$ ), thereby suggesting a context-dependent pro-immunogenic role for TGF- $\beta$.

The aforementioned indications were further confirmed by correlation of these biomarkers with medium (progression-free survival (PFS)) or long-term (OS) survival of these patients with OV such that si-NFкB responses clustered with soluble-TIM3, IFN $\gamma-P D-1-P D L 1$ axis, and CA125 to predict shorter PFS/OS of patients with OV (figure 4A and online supplemental figure S9), whereas si-IFN/ISG responses clustered with LAP/TGF- $\beta$, CCL5, CCL22, and CCL11 to predict prolonged PFS/OS of patients with OV (figure 4A and online supplemental figure S9). Remarkably, an immune-amplitude analysis for si-NFKB or si-IFN/ISG responses showed that patients with OV with prolonged survival (PFS/OS) (figure 4B,C) and reduced serum-CA125 (figure 4D,E) had a more pro-immunogenic orientation between si-IFN/ISG

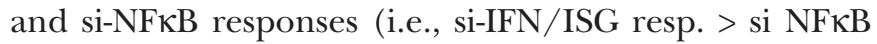
resp.), whereas patients with a clear disbalanced orientation between these two inflammatory modules (si-NFkB resp. $\geq$ si-IFN/ISG resp.) had much shorter survival (especially OS) and mostly increased serum-CA125. Overall, this established that sFIS assay-derived si-NFKB or si-IFN/ ISG responses are robust at capturing the complex and multifactorial functional immune-signaling or prognostic modules in the periphery of patients with OV.

\section{Peripheral myeloid, but not lymphoid, cells associate with si- NFKB and si-IFN/ISG responses}

The aforementioned observations suggested that si-NFKB or si-IFN/ISG responses are both linked to myeloid, rather than lymphoid, compartment. To confirm this, we correlated serum-CA125, si-NFkB, or si-IFN/ISG response and (sample-matched) absolute immune cell counts in the blood, for lymphocytes, monocytes/ myeloid cells, neutrophils, and platelets. Remarkably, serum-CA125 and si-NFKB and si-IFN/ISG responses collectively showed negative correlation with lymphocytes (figure 4F). On the level of myeloid cells, serum-CA125 and si-NFKB responses positively correlated with monocytic myeloid cells and neutrophils, whereas si-IFN/ISG responses preferred to positively correlate with monocytic myeloid cells and platelets (figure 4F). This implied the presence of a contradictory interplay between platelets and neutrophils. Herein, the disjointed trends for neutrophils or lymphocytes were intriguing since neutrophilto-lymphocyte (NLR) ratio is a well-established negative prognostic biomarker. ${ }^{5}$ Interestingly, specifically si-NFkB response (and to a certain extent, serum-CA125) positively correlated significantly to NLR (figure $4 \mathrm{G}, \mathrm{H}$ ), thereby reinforcing its negative prognostic role. However, si-IFN/ISG response failed to show correlation with NLR (figure 4I). Overall, this confirms that in OV, the peripheral immune macroenvironment exhibits disjointed myeloid and lymphoid crosstalk on the levels of si-NFkB or si-IFN/ISG responses. 

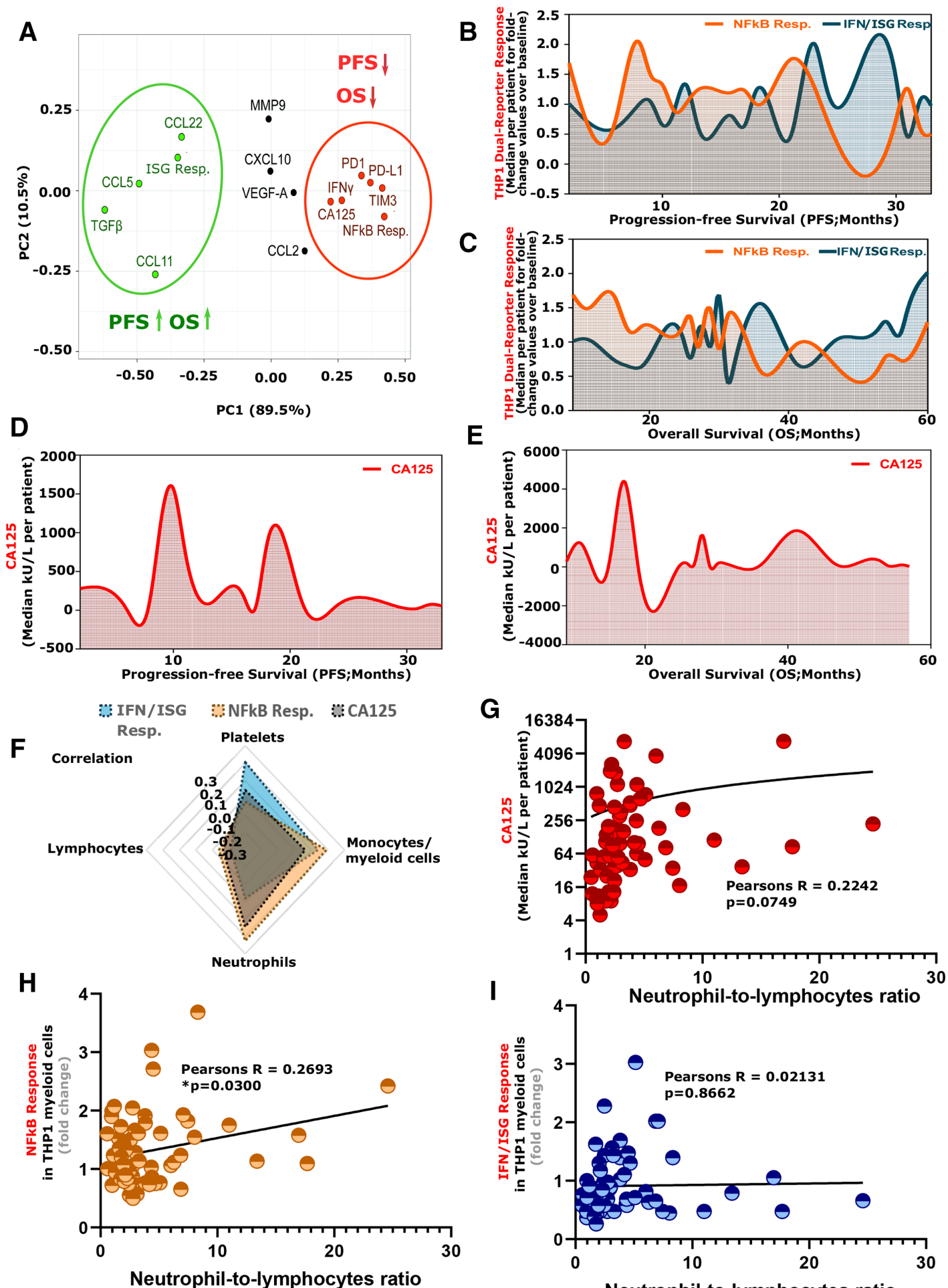

Neutrophil-to-lymphocytes ratio

Figure 4 Immunological characteristics of si-NFKB/si-IFN/ISG responses and their prognostic impact on patients with OV. (A) PCA representation of Spearman's correlation analyses between PFS or OS of patients with OV with si-NFKB and si-IFN/ ISG response, CA125 and immunological factors profiled from the serum of the UZL-CSI OV dataset (fold change to baseline/ healthy serum) (median value-based data integration for multiple serum samples per patient, $n=32$ ). (B-E) Cubic spline analyses of si-NFKB or si-IFN/ISG responses $(B, C)$ or CA125 concentration (D,E) profiled from the serum of the UZL-CSI OV cohort dataset (median value-based data integration for multiple serum samples per patient; fold change to baseline/healthy serum) and distributed as per PFS (B,D) or OS values (C-E) ( $n=32)$. (F) Radar plot of Pearson correlation values of CA125 and si-NFKB and si-IFN/ISG response with blood cell counts $(n=66)$. (G-I) Pearson correlation of CA125 (G), si-NFkB (fold change to baseline/healthy serum) $(\mathrm{H})$ and si-IFN/ISG (fold change to baseline/healthy serum) (I) responses, to NLR (CA125, $n=64$; si$\mathrm{NF \kappa B} / \mathrm{si}$-IFN/ISG response, $\mathrm{n}=65$; $\left.{ }^{*} \mathrm{p}<0.05\right)$. CA125, carbohydrate antigen 125; IFN, interferon; ISG, interferon-stimulated gene; $\mathrm{NF \kappa B}$, nuclear factor kappa-light-chain enhancer of activated B cells; NLR, neutrophil-to-lymphocyte ratio; OS, overall survival; OV, ovarian cancer; PFS, progression-free survival; si, serum-induced; UZL-CSI, UZ Leuven-Cell Stress Immunity cohort. 


\section{si-NFKB or si-IFN/ISG responses associate with differential prognosis in $\mathrm{OV}$}

Next, we wanted to estimate the clinical biomarker associations of our sFIS assay readouts. First, we pursued differential tumor-level $\mathrm{pR}$ prediction for sFIS assay readouts relative to serum-CA125 (i.e., complete response (CR), partial response, stable disease, or progressive disease (PD)). Serum-CA125 was largely reduced in patients with OV showing CR, whereas it was strongly enriched in other pR subgroups, especially PD (online supplemental figure S10A). Accordingly, non-responder patients with OV (with PD) had significantly higher serum-CA125 than responder patients with OV (with CR) (online supplemental figure S10B). However, similar differentiating trends for $\mathrm{pR}$ were absent for sFIS assay readouts (online supplemental figure S10C-F).

Serum-CA125's superiority in predicting differential $\mathrm{pR}$ is consistent with its clinical performance. ${ }^{25} 50$ However, the inability of sFIS assay readouts to not reliably predict $\mathrm{pR}$ is also in line with frequently published observations that most immunobiomarkers are better at predicting 'long-term' survival trends rather than 'short-term' $\mathrm{pR}^{51}{ }^{52}$ Accordingly, the patients with OV with shorter PFS ( $<2$ years) (figure $5 \mathrm{~A}$ ) or shorter OS $(<3$ years) (figure $5 \mathrm{~B}$ ) possessed serum that induced significantly higher NFKB responses, whereas patients with OV with longer PFS/OS (>2/3 years) (figure $5 \mathrm{C}, \mathrm{D}$ ) had higher si-IFN/ISG responses. Herein, heightened serum-CA125 partially indicated shorter PFS (figure 5E) but failed to predict differential OS (figure 5F). Accordingly, si-NFKB response was significantly associated with reduced PFS/OS of patients with OV, whereas si-IFN/ ISG responses were only significantly associated with OS, while serum-CA125 could only partially predict differential PFS but not OS (online supplemental figure S11A,B). Interestingly, similar to serum-CA125, NLR also did not correlate with PFS/OS in a conclusively negative prognostic manner (online supplemental figure S11C,D).

These trends were also supported by univariate KaplanMeier survival analysis of the PFS of patients with OV (figure 5G,H, and online supplemental figure S11E) or OS (figure 5I,J and online supplemental figure S11F), such that very high ( $>75$ th percentile cut-off) si-NFKB responses were associated with reduced survival, whereas very high si-IFN/ISG responses were associated with prolonged survival. These trends were largely substantiated by multivariate Cox Proportional Hazards (CoxPh) modeling, correcting for age, tumor stage, or intra-sFIS assay variations (online supplemental figure S12), especially for OS (online supplemental figure S12D) and differential fold-change cut-off analyses (online supplemental figure S12B-F). Overall, this highlights the biomarker efficacy of our sFIS assay (which simultaneously integrates a negative and positive prognostic factor within a single modality) in predicting medium-term/ long-term survival of patients with OV.

\section{si-NFKB responses predict malignancy risk in patients with} OV

Finally, we wished to validate as well as extend our most reliable observations in a validation cohort. Serum-CA125 is routinely used for OV diagnosis; however, there is ample room for co-diagnostic modalities. Since si-NFkB response exhibited reliable negative prognostic impact and association with pro-metastatic wound healing-like signaling, we became curious about its co-diagnostic efficacy. Hence, we retrospectively analyzed serum samples from a largescale (699 patients), multicenter European consortium biomarker study, TRANS-IOTA. ${ }^{19} 53$ TRANS-IOTA involved serum from patients recruited in Italy (Rome/ Milan), Czech Republic (Prague), Belgium (Genk/ Leuven), and UK (London). TRANS-IOTA consisted of serum collected at diagnosis from patients with benign ovarian lesions $(\mathrm{n}=404)$, borderline tumors (borderline $\mathrm{OV}, \mathrm{n}=90$ ), or invasive/metastatic tumors (malignant $\mathrm{OV}$, $\mathrm{n}=205$ ). Herein, PFS (but not OS) estimates were available for patients with malignant OV. For patient characteristics, please see online supplemental table 2).

Largely irrespective of sub-histological categories, serum-CA125 showed the highest increase in patients with malignant OV (figure 6A). Similarly, although si-NFkB response was observed in patients with benign lesions/ borderline $\mathrm{OV}$, it was more highly enriched in malignant OV (figure 6B). Overall, both serum-CA125 (figure 6C) and si-NFKB responses (figure $6 \mathrm{D}$ ) significantly differentiated malignant OV from benign lesions or borderline OV. In line with discovery cohort, si-NFkB responses consistently exceeded si-IFN/ISG responses irrespective of histopathological categories (figure $6 \mathrm{~B}$ and online supplemental figure S13A-C). Within the malignant OV category, serum-CA125 was strongly proficient at distinguishing the different tumor stages, showing a consistent ascending trend from stage I to stage IV malignant OVs (online supplemental figure S14A). However, si-NFkB response was mainly able to distinguish the most aggressive stage IV from the least aggressive stage I (online supplemental figure S14B). Overall, in line with the aforementioned trends, very high-serum CA125 or si-NF $\kappa$ B response was associated with lower PFS in patients with malignant $\mathrm{OV}$, with statistical significance achieved only by si-NFKB response (figure 6E,F). The si-IFN/ISG responses did not phenocopy above trends for si-NFkB response (online supplemental figure S15A) and, in line with our discovery cohort, si-IFN/ISG responses failed to predict differential PFS (online supplemental figure S15B). Taken together, these observations validate si-NFKB response as a negative prognostic biomarker, with putative co-diagnostic efficacy along with serum-CA125.

\section{Peripheral immunodynamic status can guide chemo- immunotherapy regime's design}

Patients with OV exhibiting a bad prognostic, si-NFkB response $^{\mathrm{HIGH}}$ si-IFN/ISG response $\mathrm{LOW}^{\mathrm{LONUL}}$ status, is consistent with OV's immunoresistant nature. Hence, we wondered whether blunting si-NFKB response can 

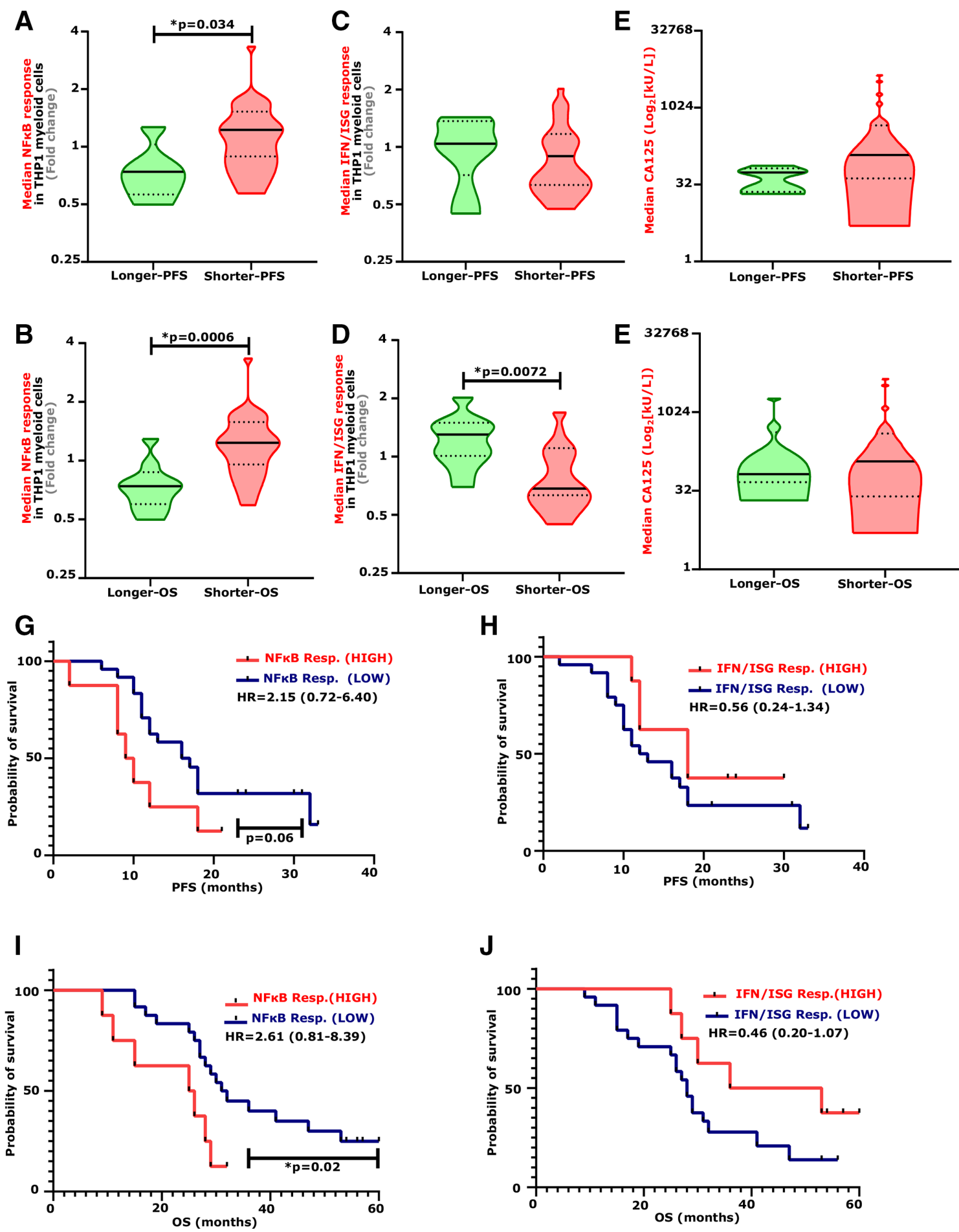

Figure 5 Prognostic impact of si-NFKB or si-IFN/ISG responses of patients with OV. (A-F) Violin plots of si-NFkB responses $(A, B)$ or si-IFN/ISG responses (C,D) and CA125 concentration (E,F) (median value-based data integration for multiple serum samples per patient) profiled from the serum of the UZL-CSI OV cohort (fold change to baseline/healthy serum). These were subdivided as longer and shorter PFS (CA125, longer PFS $n=5$ vs shorter PFS $n=26$; si-NFkB or si-IFN/ISG resp., longer PFS $n=5$ vs shorter PFS $n=27$ ) (A,C,E) or OS (CA125, longer OS $n=8$ vs shorter OS $n=23$; si-NFKB or si-IFN/ISG resp., longer PFS $n=9$ vs shorter PFS $n=23$ ) (B,D,F) based on a cut-off of 2 years of PFS or 3 years of OS (Mann-Whitney test, two-tailed; $\left.{ }^{*} \mathrm{p}<0.05\right)$. ( $\left.\mathrm{g}-\mathrm{j}\right) \mathrm{KM}$ plots of si-NFKB $(\mathrm{G}, \mathrm{I})$ or si-IFN/ISG $(\mathrm{H}, \mathrm{J})$ responses (median value-based data integration for multiple serum samples per patient) of the UZL-CSI OV cohort. Subdivision of PFS $(\mathrm{G}, \mathrm{H})$ or OS $(\mathrm{I}, \mathrm{J})$ as high or low were based on the 75th percentile cut-off (si-NFKB or si-IFN/ISG resp. vs PFS/OS, HIGH $n=8$ vs low $n=24$ ). The plots depict the $\mathrm{HR} \pm 95 \% \mathrm{Cl}$ (log-rank Mantel-Cox test, $\left.{ }^{*} \mathrm{p}<0.05\right)$. CA125, carbohydrate antigen 125; IFN, interferon; ISG, interferon-stimulated gene; KM, KaplanMeier; NFKB, nuclear factor kappa-light-chain enhancer of activated B cells; OS, overall survival; OV, ovarian cancer; PFS, progression-free survival; si, serum-induced; UZL-CSI, UZ Leuven-Cell Stress Immunity cohort. 

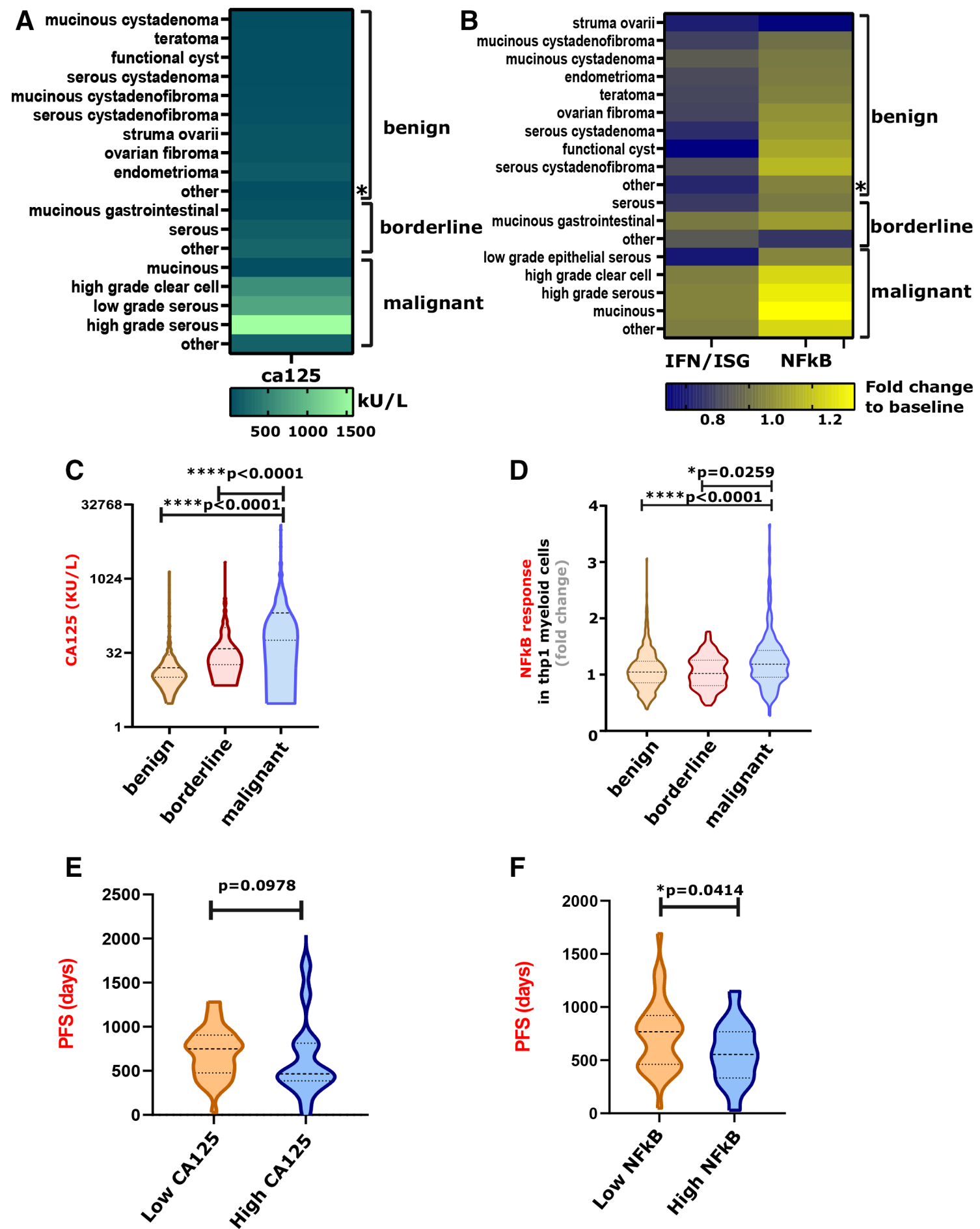

Figure 6 sFIS assay testing to estimate malignancy risk in a validation cohort of patients with ovarian cancer. (A,B) Heatmap representation of CA125 (A) or si-NFKB and si-IFN/ISG response (B) per histology category profiled from the serum of the TRANS-IOTA cohort (fold change to baseline/healthy serum, averaged values; for full patient details and numbers, see online supplemental table 2). " ${ }^{*}$ indicates inclusion of patients with cysts at the ultrasound. (C,D) Violin plot of CA125 (C) or si-NFkB

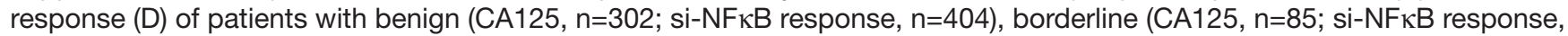
$\mathrm{n}=90$ ) and malignant (CA125, $\mathrm{n}=198$; si-NFKB response, $\mathrm{n}=205)$ cancers, profiled from the serum of the TRANS-IOTA cohort (fold change to baseline/healthy serum, Kolmogorov-Smirnov test; * $\mathrm{p}<0.05$ ). (E,F) Violin plot of PFS based on CA125 (low, $n=42$; high, $n=14$ ) (E) or si-NFKB response (low, $n=41$; high, $n=15)(F)$ of patients with cancer (fold change to baseline/healthy serum) (Mann-Whitney test, $\left.{ }^{*} \mathrm{p}<0.05\right)$. Subdivision of CA125 and si-NFKB $(\mathrm{E}, \mathrm{F})$ as high or low were based on the 75 th percentile cutoff. CA125, carbohydrate antigen 125; IFN, interferon; ISG, interferon-stimulated gene; NFkB, nuclear factor kappa-light-chain enhancer of activated B cells; PFS, progression free survival; si, serum-induced; sFIS, serum-functional immunodynamic status. 


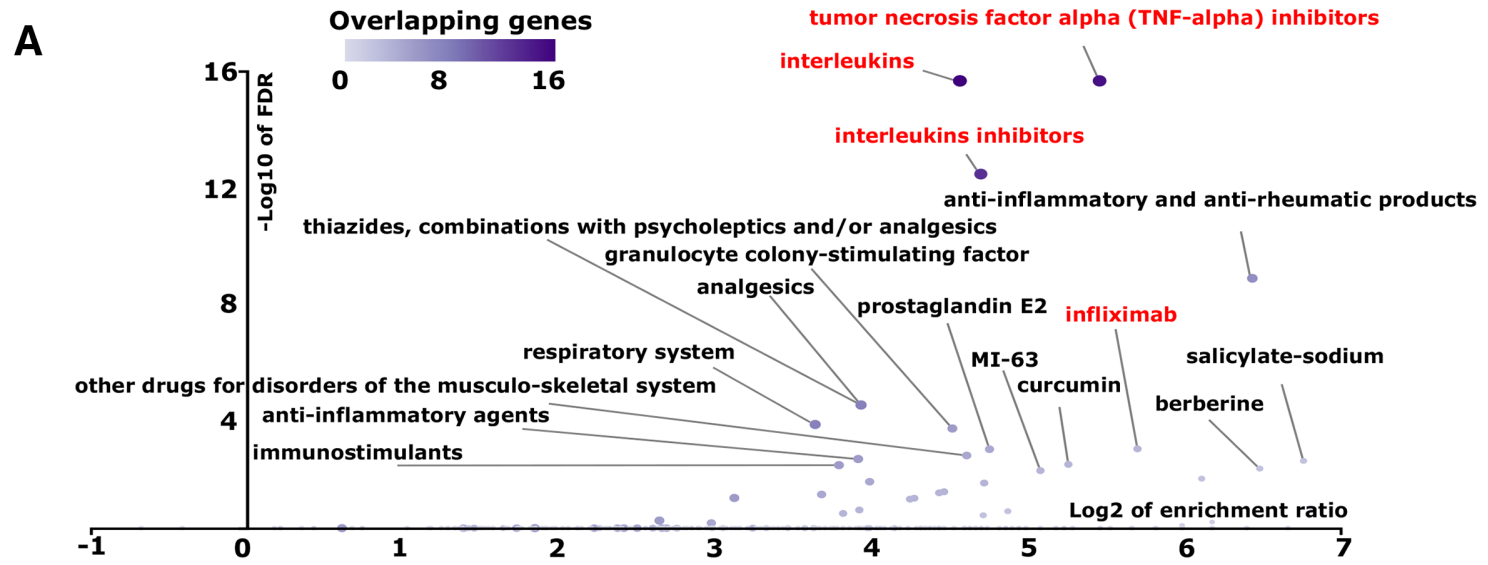

B

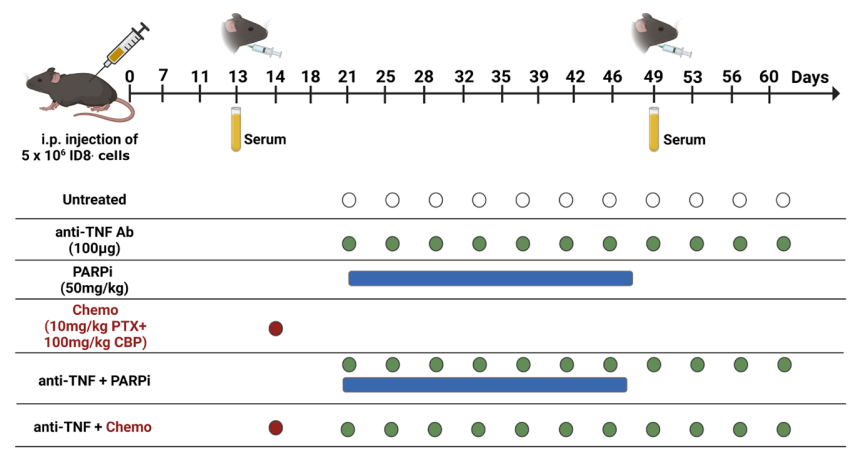

C $\quad 3774$ Dual-Reporter activity

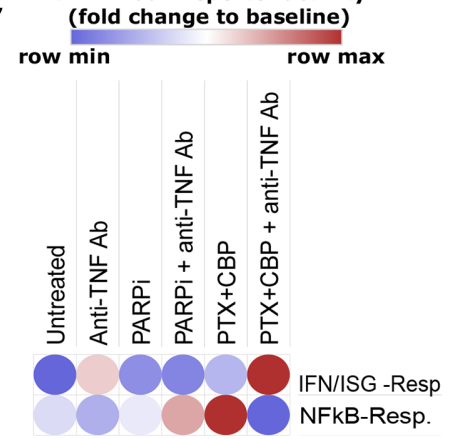

D

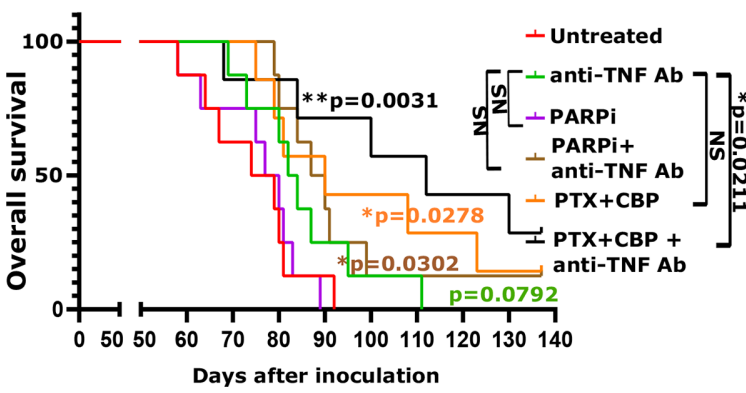

E

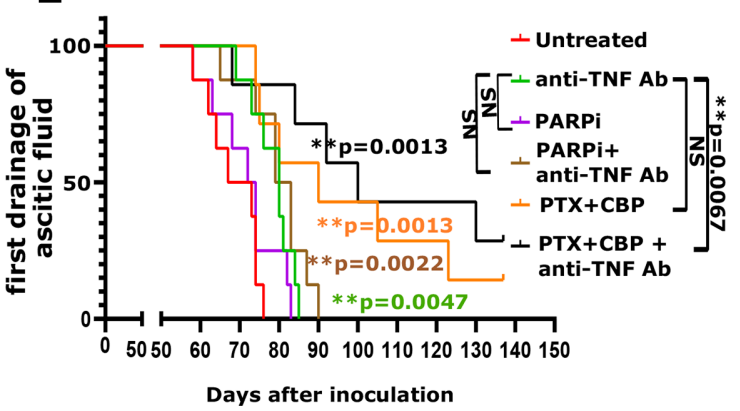

Figure 7 sFIS assay-based prediction of chemoimmunotherapy regime's design and in vivo testing in murine metastatic ovarian cancer model. (A) In silico drug-prediction analyses based on the NFKB response signature (cut-offs: multiple adjustment test, Bonferroni; significance level, adjusted $p$ value $=0.01$ ). Colors indicate overlapping genes between NFkB response signature and literature-associated drug-gene sets. (B) Overview of the tumor inoculation, serum collection, and therapeutic treatment schedules for the mice experiments. (C) si-IFN/ISG and si-NFKB response of J774 dual-reporter cell lines exposed to mouse serum obtained from day 49 as a ratio to day 13 (control, $n=8$; anti-TNF Ab, n=8; PARPi, $n=7$; PARPi and anti-TNF Ab, n=8; PTX-CBP, n=7; PTX-CBP and anti-TNF Ab, n=6). (D,E) Kaplan-Meier plots of overall survival (D) or survival while considering the first drainage of ascitic fluid (E) of metastatic ID8 tumor-bearing mice treated with different therapy regimes (control, $n=8$; anti-TNF Ab, $n=8$; PARPi, $n=8$; PARPi and anti-TNF Ab, $n=8$; PTX-CBP, $n=7$; PTX-CBP and anti-TNF Ab, $\mathrm{n}=7$ ) (log-rank Mantel-Cox test; ${ }^{\star} \mathrm{p}<0.05$, ${ }^{* \star} \mathrm{p}<0.01$ ). IFN, interferon; ISG, interferon-stimulated gene; NFkB, nuclear factor kappalight-chain enhancer of activated B cells; PARPi, PARP inhibitor; PTX-CBP, paclitaxel +carboplatin; sFIS, serum-functional immunodynamic status.

be used as a guiding strategy for precision anti-OV immunotherapy. To address this, we pursued an in silico drug prediction relying on a computational algorithm exploiting biomedical literature-associated drug-gene relationships to predict drugs or drug-target's associations to the NFKB response signature (figure 7A). These analyses enriched for anti-cytokine immunotherapies or inflammatory therapeutic targets, especially TNF inhibitory/blocking therapeutics like TNF-alpha inhibitors or infliximab (an anti-TNF antibody $(\mathrm{Ab})$ ) (figure 7). This was concurrent with the observation that, among various cytokines we screened, TNF was predictably the most robust $\mathrm{NF \kappa B}$ response inducer (figure 2D).

The notion of applying anti-cytokine immunotherapies like anti-TNF Ab is not new. While such anti-cytokine immunotherapies have shown preclinical success, they 
have often failed in clinical trials. ${ }^{4}$ It has been proposed that their success might be contingent on biomarkerdriven application; however, such precision biomarkers are elusive. ${ }^{4}$ Therefore, we wondered whether si-NFкB response can better guide application of anti-TNF $\mathrm{Ab}$ in patients with OV. In fact, reanalyses of a small (only existing) human OV clinical trial administering infliximab, wherein pretreatment/on-treatment transcriptome for $\mathrm{OV}$ ascites was available, ${ }^{545}$ showed that $T N F$ in $\mathrm{OV}$ ascites could not differentiate infliximab-responding versus non-responding patients (online supplemental figure $\mathrm{S} 15 \mathrm{C}$ ), while elevated $\mathrm{NF \kappa B}$ response signature differentiated them relatively better (online supplemental figure S15D).

To validate this via sFIS assay, we used the murine ID8 cell-based orthotopic model of metastatic $\mathrm{OV}^{56}$ since these metastatic ID8 tumor-bearing mice experience a latent spike in serum TNF levels (online supplemental figure S15E). Various studies have demonstrated antitumor and immunostimulatory impact of anti-OV therapies like paclitaxel + carboplatin (PTX-CBP) and/or PARP inhibitors (PARPi) ${ }^{5758}$ yet it is not clear which of these therapies might best potentiate the antitumor efficacy of anti-TNF Ab. Also, we were curious whether sFIS assay can predict the antitumor efficacy of the aforementioned therapeutics (alone or in combination with anti-TNF Ab). Hence, we treated the ID8 tumor-bearing mice with PTX-CBP, PARPi, or anti-TNF Ab, or combinations thereof (figure $7 \mathrm{~B}$ ). Initially, we collected the murine serum at baseline and during the aforementioned treatments (figure 7B) and screened the ability of these serum samples to induce si-NFKB or si-IFN/ISG responses in a murine version of our sFIS assay, that is, J774 murine myeloid cells stably expressing two inducible reporter constructs for NFאB or IFN/ISG response signaling. Interestingly, a ratio-metric analysis (on to pretreatment ratio) of murine sFIS assay derived si-NFKB or si-IFN/ISG responses showed that anti-TNF Ab exhibited threshold tendencies to reduce si-NFKB responses while marginally increasing si-IFN/ISG responses (figure 7C). Interestingly, combining anti-TNF $\mathrm{Ab}$ with PTX-CBP created a much better peripheral pro-immunogenic status (si-NFKB response ${ }^{\mathrm{LOW} \mathrm{si}-\mathrm{IFN} \text { / }}$ ISG response ${ }^{\mathrm{HIGH}}$ ) than combining with PARPi (si-NFkB response $^{\mathrm{MED}} \mathrm{si}-\mathrm{IFN} / \mathrm{ISG}$ response ${ }^{\mathrm{LOW}}$ ), or either therapy alone (figure $7 \mathrm{C}$ ).

To understand whether sFIS assay can predict antitumor efficacy in vivo, we estimated two long-term survival parameters, that is, OS and OS relative to first ascitic fluid's drainage (OS-AFD). The long-term OS trends indeed followed the aforementioned sFIS assay predictions, such that the ID8 tumor-bearing mice treated with PTX-CBP and anti-TNF Ab triple-combo had the longest OS (figure 7D) and OS-AFD (figure 7E). In fact, PTX$\mathrm{CBP}$ and anti-TNF Ab combo was the only condition that had significantly higher anti-OV efficacy than anti-TNF Ab alone (figure $7 \mathrm{D}, \mathrm{E}$ ), thereby highlighting the superiority of PTX-CBP (over PARPi) in unleashing anti-TNF Ab's potential. Moreover, a ratio-metric analyses established that a peripheral si-IFN/ISG response ${ }^{\mathrm{HIGH}}$ si-NFкB response $\mathrm{e}^{\mathrm{LOW}}$ status positively correlated with prolonged OS (online supplemental figure S15F). In conclusion, the sFIS assay may help in designing anti-OV regimen, wherein si-IFN/ISG response ${ }^{\mathrm{HIGH}}$ si-NFkB response $\mathrm{L}^{\mathrm{LOW}}$ phenotype could guide selection of pro-immunogenic therapies.

\section{DISCUSSION}

Through a data-driven in silico framework driven by cancer patient's data, we conceptualized a first-inclass, non-invasive, in sitro sFIS assay for capturing the si-NFKB or si-IFN/ISG responses of patients with OV. The sFIS assay was able to capture the peripheral immunodynamics of patients with OV better than individual serum-linked cytokines, chemokines, or soluble immune checkpoints. Nevertheless, both approaches of serum screening altogether established that a hyperinflammatory $\mathrm{NFKB}$ signaling dominates the periphery of patients with OV, with strong depletion of si-IFN/ISG responses. Herein, high si-NFKB response was associated with shortened PFS/OS, while si-IFN/ISG response was associated with prolonged OS. This culminated into a patient immunostratification indicating low OS for OV patients with si-NFKB response ${ }^{\mathrm{HIGH}}$ Si-IFN/ISG response ${ }^{\mathrm{LOW}}$ status and high OS for those with si-NFKB response ${ }^{\text {LOW }}$ si-IFN/ISG response $\mathrm{HIGH}^{\mathrm{HIG}}$. Besides, the malignant/invasive nature of $\mathrm{OV}$ was distinguished by a serum-CA1 $25^{\mathrm{HIGH}} \mathrm{si}-\mathrm{NF \kappa B}$ response ${ }^{\mathrm{HIGH}}$ si-IFN/ISG response ${ }^{\mathrm{LOW}}$ status, such that si-NFKB response was highest in patients with invasive/ malignant ovarian lesions, as compared with benign or borderline lesions. This provides support to the notion that tumor aggression associates with systemic inflammatory remodeling. ${ }^{6}$ Finally, we were also able to successfully use a murine version of our sFIS assay in preclinical settings of metastatic ID8 tumor model to predict and confirm the superior proficiency of chemo-immunotherapy regime of PTX-CBP and anti-TNF Ab in achieving a favorable peripheral si-NFkB response ${ }^{\mathrm{LOW}}$ si-IFN/ISG response ${ }^{\mathrm{HIGH}}$ status and efficaciously controlling OV. Altogether these observations exemplify the multifaceted utility of the sFIS assay in copredicting malignancy risk, differential survival benefit and rationale designing of immunotherapeutic regimen.

The sFIS assay approach (along with multiplex cytokine/immune-factor screening, blood immune cell counts, and computational immunology) also allowed us to gain some crucial insights into the peripheral immunobiology of patients with OV. For instance, si-NFkB responses mainly aligned with wound healing-like prometastatic signaling, while si-IFN/ISG responses positively correlated with serum-LAP/TGF- $\beta$ (rather than serum IFN- $\alpha / \gamma$ or CXCL10). Herein, high serum-LAP/TGF- $\beta$ levels were also associated with prolonged OS/PFS, such that high TGF- $\beta$ concentrations directly induced IFN/ ISG responses, thereby revealing an unanticipated proimmunogenic activity for TGF- $\beta .^{59}$ Remarkably, in the 
murine metastatic ID8 tumor model, combining anti-TNF Ab with PTX-CBP chemotherapy unleashed si-IFN/ISG responses, thereby implying a role for pro-tumorigenic NFKB signaling in blunting IFN/ISG responses. Overall, this substantiates a disbalance between these two pathways as a major source of immune disparities in patients with OV. Since OV is a highly immunosuppressive and ICBresistant tumor, ${ }^{60}$ there is an urgent need to introduce novel anti-OV immunotherapies, possibly prioritized based on their ability to potentiate si-IFN/ISG responses while blunting si-NFKB responses. Thus, anti-OV 'immunogenic' interventions ${ }^{61}$ guided by sFIS assay could be used to improve the outlook for patients with OV.

The sFIS assay also offers several practical advantages, for example, its 96-well plate-based throughput nature and reliance on non-invasive serum samples. Also, a major strength of our approach is that we could use publicly available OV multiomics data to predict peripheral immunodynamic status based on NFKB or IFN/ISG responses, which was largely confirmed via sFIS assay. This allows the usage of NFKB and ISG response genetic signatures as companion transcriptomic biomarkers for tumor analyses that closely complement our sFIS assay. Finally, the possibility to guide or design chemo-immunotherapy regimen via sFIS assay, as implied by our in vivo experiments, opens doors to many novel possibilities. Nevertheless, there are also some limitations of our study that require future attention; for example, a prospective, highpowered, randomized clinical trial is necessary to fully validate the serum-driven prediction efficacy of the sFIS assay. We believe such a trial should include longitudinal serum sampling (i.e, pre-treatment and post-treatment) in a multiarm comparative treatment setting (e.g., immunotherapy vs chemotherapy). OV should be prioritized for such a trial based on the above data; however, based on TCGA analyses, CESC, HNSC or LIHC might also be good indications to test the contrasting impact of si-NFKB versus si-IFN/ISG responses with sFIS assay.

In conclusion, we believe that our sFIS assay may facilitate personalized patient monitoring, immunostratification and immunotherapeutic decision-making. The differential si-NFkB or si-IFN/ISG response can shed light on how the peripheral immune responses are regulated or dysregulated. Thus, our study serves as a proof of concept for further clinical development of peripheral immunodynamic biomarkers.

\section{Author affiliations}

${ }^{1}$ Laboratory of Cell Stress \& Immunity, Department of Cellular \& Molecular Medicine, KU Leuven, Leuven, Belgium

${ }^{2}$ Department of Oncology, Leuven Cancer Institute, Laboratory of Tumor Immunology and Immunotherapy, KU Leuven, Leuven, Belgium

${ }^{3}$ Department of Oncology, Leuven Cancer Institute, Laboratory of Gynaecologic Oncology, KU Leuven, Leuven, Belgium

${ }^{4} \mathrm{JJP}$ Biologics, Warsaw, Poland

${ }^{5}$ Department of Development and Regeneration, KU Leuven, Leuven, Belgium

${ }^{6}$ Queen Charlotte's and Chelsea Hospital, Imperial College, London, UK

${ }^{7}$ Dipartimento Scienze della Salute della Donna e del Bambino, Fondazione

Policlinico Universitario A. Gemelli, Istituto di Ricovero e Cura a Carattere Scientifico, Rome, Italy
${ }^{8}$ Dipartimento Scienze della Vita e Sanità pubblica, Università Cattolica del Sacro Cuore, Rome, Italy

${ }^{9}$ Charles University, Praha, Czech Republic

${ }^{10}$ Ziekenhuis Oost-Limburg, Genk, Belgium

${ }^{11}$ National Cancer Institute of Milan, Milan, Italy

${ }^{12}$ Department of Gynaecology and Obstetrics, UZ Leuven, Leuven, Belgium

${ }^{13}$ Department of Microbiology, Immunology and Transplantation, Laboratory of

Virology and Chemotherapy, Rega Institute, KU Leuven, Leuven, Belgium

${ }^{14}$ Department of Cellular and Molecular Medicine, Cell Death Research and Therapy Laboratory, KU Leuven, Belgium

${ }^{15}$ VIB Center for Cancer Biology, VIB, Leuven, Belgium

${ }^{16}$ Laboratory for Molecular Digestive Oncology, Department of Oncology, KU Leuven, Leuven, Belgium

\section{Twitter Abhishek D Garg @AbhishekDGarg}

Acknowledgements We thank Gitte Thirion, Katja Vandenbrande and Sandra Claes for their excellent assistance with the in vivo experiments and LUMINEX assay. We thank Dr Stefan Naulaerts (UCL, Brussels), Professor Stephanie Humblet-Baron (KU Leuven), and Dr Sigrid Hatse (KU Leuven) for attentively reading the manuscript and providing valuable suggestions, and Dr Naulaerts for additional feedback on statistics.

Contributors JS performed the serum-functional immunodynamic-status assay and associated data handling and analyses. AV and RW performed the mice experiments. IV, RL, PA, and YB helped with serum sample handling and/or sFIS assay. TBa, CL, AT, DF, CVH, TBo, VC, WF, DT, IV, and AC helped with patient serum collection, handling, and processing. DS helped with Luminex data analyses and collection. LB helped with anti-tumor necrosis factor antibody analyses. DB and $A D G$ performed all the bioinformatics analyses. $S T, A C$, and $A D G$ provided senior supervision and critical revision of the manuscript. AC provided supervision on patient and murine sample handling. ADG was the lead investigator of the project and coordinated the overall project, supervised the in silico, in vitro or in vivo research designs, as well as wrote the manuscript. ADG is responsible for the overall content as the guarantor.

Funding This study is supported by Research Foundation Flanders (FWO) (Fundamental Research Grant, G0B4620N to ADG; G0B4716N to DT; Excellence of Science/EOS grant, 30837538, for 'DECODE' consortium, for ADG, PA), KU Leuven (C1 grant, C14/19/098, C3 grant, C3/21/037, and POR award funds, POR/16/040 to ADG; C24/15/037 to DT), Kom op Tegen Kanker (Stand Up To Cancer, the Flemish Cancer Society) (KOTK/2018/11509/1 to ADG and PA; KOTK/2016/10728/2603 to AC, DT, and IV; KOTK/2019/11955/1 to AC, ADG, IV, and 11758 to AK), Amgen Chair for Therapeutic Advances in Ovarian Cancer (2017/LUF/00069 to IV), Vriendtjes Tegen Kanker (2016_LUF_00078 to IV) and VLIR-UOS (iBOF grant, iBOF/21/048, for 'MIMICRY' consortium to ADG and ST). IsVa is supported by FWO-SB PhD Fellowship (1S06821N). CL was supported by the Linbury Trust Grant LIN2600. DMB is supported by KU Leuven's Postdoctoral mandate grant (PDMT1/21/032), and the Belgian Federation against Cancer grant numbers 2018-127 and 2016-133 and by a grant from Fondation Roi-Baudouin to ST. ST and DT are further supported by a Senior Clinical Investigator award of FWO.

Competing interests The sFIS assay is currently the subject of an ongoing PCT patent application. ADG has received consulting/advisory/lecture honoraria from Boehringer Ingelheim and Miltenyi Biotec.

\section{Patient consent for publication Not applicable.}

Ethics approval Clinical patient studies were approved by the local ethics committee of UZ Leuven, Belgium (s51375 and s59207, for TRANS-IOTA; s56311, s50887 and s64035 for OV-IMM-2014/umbrella). UZ Leuven was the coordinating ethical committee for TRANS-IOTA. Preclinical mice experiments performed were in line with the Belgian (Royal Decree, May 29, 2013), Flemish (Decision of the Flemish Government to adapt the Royal Decree of May 29, 2013, February 17, 2017) and European (Directive 2010/63/EU) regulations on the protection of animals used for scientific purposes. Approval of the local ethical committee at KU Leuven, Belgium was obtained (P125/2017) for all mice experiments.

Provenance and peer review Not commissioned; externally peer reviewed.

Data availability statement Some of the data are available in a public, open access repository (e.g., TCGA, GEO or scRNAseq datasets). Those data not available in such repositories, are available upon reasonable request.

Supplemental material This content has been supplied by the author(s). It has not been vetted by BMJ Publishing Group Limited (BMJ) and may not have been 
peer-reviewed. Any opinions or recommendations discussed are solely those of the author(s) and are not endorsed by BMJ. BMJ disclaims all liability and responsibility arising from any reliance placed on the content. Where the content includes any translated material, BMJ does not warrant the accuracy and reliability of the translations (including but not limited to local regulations, clinical guidelines, terminology, drug names and drug dosages), and is not responsible for any error and/or omissions arising from translation and adaptation or otherwise.

Open access This is an open access article distributed in accordance with the Creative Commons Attribution Non Commercial (CC BY-NC 4.0) license, which permits others to distribute, remix, adapt, build upon this work non-commercially, and license their derivative works on different terms, provided the original work is properly cited, appropriate credit is given, any changes made indicated, and the use is non-commercial. See http://creativecommons.org/licenses/by-nc/4.0/.

\section{ORCID iDs}

Isaure Vanmeerbeek http://orcid.org/0000-0001-5894-6008

Yani Berckmans http://orcid.org/0000-0003-1350-5596

An Coosemans http://orcid.org/0000-0002-7321-4339

Abhishek D Garg http://orcid.org/0000-0002-9976-9922

\section{REFERENCES}

1 Nalejska E, Mączyńska E, Lewandowska MA. Prognostic and predictive biomarkers: tools in personalized oncology. Mol Diagn Ther 2014;18:273-84.

2 Califf RM. Biomarker definitions and their applications. Exp Biol Med 2018;243:213-21.

3 Vanmeerbeek I, Borras DM, Sprooten J, et al. Early memory differentiation and cell death resistance in $\mathrm{T}$ cells predicts melanoma response to sequential anti-CTLA4 and anti-PD1 immunotherapy. Genes Immun 2021;22:108-19.

4 Hou J, Karin M, Sun B. Targeting cancer-promoting inflammation have anti-inflammatory therapies come of age? Nat Rev Clin Oncol 2021;18:261-79.

5 Hiam-Galvez KJ, Allen BM, Spitzer MH. Systemic immunity in cancer. Nat Rev Cancer 2021;21:345-59.

6 Chechlinska M, Kowalewska M, Nowak R. Systemic inflammation as a confounding factor in cancer biomarker discovery and validation. Nat Rev Cancer 2010;10:2-3.

7 Bassez A, Vos H, Van Dyck L, et al. A single-cell map of intratumoral changes during anti-PD1 treatment of patients with breast cancer. Nat Med 2021;27:820-32.

8 Ferreira CG, Nicolini A, Dalurzo L, et al. The value of biomarkers in optimizing the use of Immuno-oncologic therapy. Curr Drug Targets 2018;20:81-6.

9 Mehnert JM, Monjazeb AM, Beerthuijzen JMT, et al. The challenge for development of valuable Immuno-oncology biomarkers. Clin Cancer Res 2017;23:4970-9.

10 Gnjatic S, Bronte V, Brunet LR, et al. Identifying baseline immunerelated biomarkers to predict clinical outcome of immunotherapy. $\mathrm{J}$ Immunother Cancer 2017;5:44.

11 Chalabi M, Fanchi LF, Dijkstra KK, et al. Neoadjuvant immunotherapy leads to pathological responses in MMR-proficient and MMRdeficient early-stage colon cancers. Nat Med 2020;26:566-76.

12 Versluis JM, Long GV, Blank CU. Learning from clinical trials of neoadjuvant checkpoint blockade. Nat Med 2020;26:475-84.

13 Knific T, Fishman D, Vogler A, et al. Multiplex analysis of 40 cytokines do not allow separation between endometriosis patients and controls. Sci Rep 2019;9:16738.

14 Krzystek-Korpacka M, Diakowska D, Kapturkiewicz B, et al. Profiles of circulating inflammatory cytokines in colorectal cancer (CRC), high cancer risk conditions, and health are distinct. Possible implications for CRC screening and surveillance. Cancer Lett 2013;337:107-14.

15 Hu-Lieskovan S, Bhaumik S, Dhodapkar K, et al. SITC cancer immunotherapy resource document: a compass in the land of biomarker discovery. J Immunother Cancer 2020;8:e000705.

16 Murphy K, Weaver C. Janeway's Immunobiology. 9th edn. New York, NY: Garland Science/Taylor \& Francis: Garland Science, 2016.

17 Kaskas NM, Moore-Medlin T, McClure GB, et al. Serum biomarkers in head and neck squamous cell cancer. JAMA Otolaryngol Head Neck Surg 2014;140:5-11.

18 Adelaja A, Taylor B, Sheu KM, et al. Six distinct NFкB signaling codons convey discrete information to distinguish stimuli and enable appropriate macrophage responses. Immunity 2021;54:916-30.

19 Coosemans A, Baert T, Ceusters J, et al. Myeloid-derived suppressor cells at diagnosis may discriminate between benign and malignant ovarian tumors. Int J Gynecol Cancer 2019;29:1381-8.
20 Landolfo C, Achten ETL, Ceusters J, et al. Assessment of protein biomarkers for preoperative differential diagnosis between benign and malignant ovarian tumors. Gynecol Oncol 2020;159:811-9.

21 Lin W-W, Karin M. A cytokine-mediated link between innate immunity, inflammation, and cancer. J Clin Invest 2007;117:1175-83.

22 Zhao H, Wu L, Yan G, et al. Inflammation and tumor progression: signaling pathways and targeted intervention. Sig Transduct Target Ther 2021;6:263.

23 Sprooten J, Garg AD. Type I interferons and endoplasmic reticulum stress in health and disease. Int Rev Cell Mol Biol 2020;350:63-118.

24 Elsworth B, Dawe K, Vincent EE, et al. MELODI: mining enriched literature objects to derive intermediates. Int J Epidemiol 2018.

25 Gadducci A, Cosio S, Tana R, et al. Serum and tissue biomarkers as predictive and prognostic variables in epithelial ovarian cancer. Crit Rev Oncol Hematol 2009;69:12-27.

26 Tian B, Nowak DE, Jamaluddin M, et al. Identification of direct genomic targets downstream of the nuclear factor- $\kappa \mathrm{B}$ transcription factor mediating tumor necrosis factor signaling. J Biol Chem 2005;280:17435-48.

27 Moserle L, Indraccolo S, Ghisi M, et al. The side population of ovarian cancer cells is a primary target of IFN- $\alpha$ antitumor effects. Cancer Res 2008;68:5658-68.

28 Hecker M, Hartmann C, Kandulski O, et al. Interferon-beta therapy in multiple sclerosis: the short-term and long-term effects on the patients' individual gene expression in peripheral blood. Mol Neurobiol 2013;48:737-56.

29 Sana T, Janatpour M, Sathe M, et al. Microarray analysis of primary endothelial cells challenged with different inflammatory and immune cytokines. Cytokine 2005;29:256-69.

30 Rusinova I, Forster S, Yu S, et al. Interferome v2.0: an updated database of annotated interferon-regulated genes. Nucleic Acids Res 2013;41:D1040-6.

31 Sprooten J, Agostinis P, Garg AD. Type I interferons and dendritic cells in cancer immunotherapy. Int Rev Cell Mol Biol 2019;348:217-62.

32 Wu TD, Madireddi S, de Almeida PE, et al. Peripheral T cell expansion predicts tumour infiltration and clinical response. Nature 2020;579:274-8.

33 Yarchoan M, Hopkins A, Jaffee EM. Tumor mutational burden and response rate to PD-1 inhibition. N Engl J Med 2017;377:2500-1.

34 Duraiswamy J, Freeman GJ, Coukos G. Therapeutic PD-1 pathway blockade augments with other modalities of immunotherapy T-cell function to prevent immune decline in ovarian cancer. Cancer Res 2013;73:6900-12.

35 Pahl HL. Activators and target genes of Rel/NF- $\mathrm{KB}$ transcription factors. Oncogene 1999;18:6853-66.

36 Boston University. NF-kB transcription factors. Available: https:// www.bu.edu/nf-kb/ [Accessed Sep 2019].

37 Ueland FR. A perspective on ovarian cancer biomarkers: past, present and yet-to-come. Diagnostics 2017;7:14

38 Qian J, Olbrecht S, Boeckx B, et al. A pan-cancer blueprint of the heterogeneous tumor microenvironment revealed by single-cell profiling. Cell Res 2020;30:745-62.

39 Wang Q, Zhang J, Tu H, et al. Soluble immune checkpoint-related proteins as predictors of tumor recurrence, survival, and T cell phenotypes in clear cell renal cell carcinoma patients. J Immunother Cancer 2019;7:334.

40 Henry NL, Hayes DF. Cancer biomarkers. Mol Oncol 2012;6:140-6.

41 Simon RM, Paik S, Hayes DF. Use of archived specimens in evaluation of prognostic and predictive biomarkers. J Natl Cancer Inst 2009;101:1446-52.

42 Owyong M, Chou J, van den Bijgaart RJ, et al. MMP9 modulates the metastatic cascade and immune landscape for breast cancer antimetastatic therapy. Life Sci Alliance 2019;2:e201800226.

43 Vandooren J, Van den Steen PE, Opdenakker G. Biochemistry and molecular biology of gelatinase $B$ or matrix metalloproteinase-9 (MMP-9): the next decade. Crit Rev Biochem Mol Biol 2013;48:222-72.

44 Koivisto L, Heino J, Häkkinen L, et al. Integrins in wound healing. Adv Wound Care 2014:3:762-83.

45 Agresti R, Triulzi T, Sasso M, et al. Wound healing fluid reflects the inflammatory nature and aggressiveness of breast tumors. Cells 2019;8:181

46 Mazzocca A, Dituri F, De Santis F, et al. Lysophosphatidic acid receptor LPAR6 supports the tumorigenicity of hepatocellular carcinoma. Cancer Res 2015;75:532-43.

47 Popnikolov NK, Dalwadi BH, Thomas JD, et al. Association of autotaxin and lysophosphatidic acid receptor 3 with aggressiveness of human breast carcinoma. Tumor Biol 2012;33:2237-43. 
48 Qing J, Liu C, Choy L, et al. Transforming growth factor $\beta /$ Smad3 signaling regulates IRF-7 function and transcriptional activation of the beta interferon promoter. Mol Cell Biol 2004;24:1411-25.

49 Pokharel SM, Shil NK, Bose SAutophagy, TGF- $\beta$, and SMAD-2/3 signaling regulates interferon- $\beta$ response in respiratory syncytial virus infected macrophages. Front Cell Infect Microbiol 2016;6:174.

50 Baert T, Van Camp J, Vanbrabant L, et al. Influence of CA125, platelet count and neutrophil to lymphocyte ratio on the immune system of ovarian cancer patients. Gynecol Oncol 2018;150:31-7.

51 Nixon AB, Schalper KA, Jacobs I, et al. Peripheral immune-based biomarkers in cancer immunotherapy: can we realize their predictive potential? J Immunother Cancer 2019;7:325.

52 Sprooten J, De Wijngaert P, Vanmeerbeerk I, et al. Necroptosis in immuno-oncology and cancer immunotherapy. Cells 2020;9:1823.

53 Kaijser J, Bourne T, Valentin L, et al. Improving strategies for diagnosing ovarian cancer: a summary of the International ovarian tumor analysis (iota) studies. Ultrasound Obstet Gynecol 2013;41:9-20.

54 Kulbe H, Chakravarty P, Leinster DA, et al. A dynamic inflammatory cytokine network in the human ovarian cancer microenvironment. Cancer Res 2012;72:66-75.
55 Charles KA, Kulbe H, Soper R, et al. The tumor-promoting actions of TNF- $\alpha$ involve TNFR1 and IL-17 in ovarian cancer in mice and humans. J Clin Invest 2009;119:3011-23.

56 Baert T, Vankerckhoven A, Riva M, et al. Myeloid derived suppressor cells: key drivers of immunosuppression in ovarian cancer. Front Immunol 2019;10:1273.

57 Lampert EJ, Zimmer A, Padget M, et al. Combination of PARP inhibitor olaparib, and PD-L1 inhibitor Durvalumab, in recurrent ovarian cancer: a proof-of-concept phase II study. Clin Cancer Res 2020;26:4268-79.

58 Lau TS, Chan LKY, Man GCW, et al. Paclitaxel induces immunogenic cell death in ovarian cancer via TLR4/IKK2/SNARE-Dependent exocytosis. Cancer Immunol Res 2020;8:1099-111.

59 French R, Feng Y, Pauklin S. Targeting TGF $\beta$ signalling in cancer: toward context-specific strategies. Trends in Cancer 2020;6:538-40.

60 Odunsi K. Immunotherapy in ovarian cancer. Ann Oncol. 2017;28:viii1-7.

61 Baert T, Garg AD, Vindevogel EVA, et al. In vitro generation of murine dendritic cells for cancer immunotherapy: an optimized protocol. Anticancer Res 2016;36:5793-802. 